\title{
The effect of alkyl substitution on the oxidative metabolism and mutagenicity of phenanthrene
}

\author{
Danlei Wang ${ }^{1}\left(\mathbb{0} \cdot\right.$ Viktoria Schramm $^{1} \cdot$ Jeroen Pool ${ }^{1} \cdot$ Eleni Pardali ${ }^{1}$ Annemarijn Brandenburg ${ }^{1}$. \\ Ivonne M. C. M. Rietjens ${ }^{1} \cdot$ Peter J. Boogaard'
}

Received: 26 November 2021 / Accepted: 27 January 2022 / Published online: 19 February 2022

(c) The Author(s) 2022

\begin{abstract}
Alkyl-substituted PAHs may be present in certain petroleum-derived products and in the environment and may eventually end up in consumer products, such as foodstuffs, cosmetics and pharmaceuticals. Safety concerns over possible exposure to alkylated PAHs have emerged. Bioactivation is a prerequisite for the mutagenicity and carcinogenicity of PAHs and has been extensively studied for non-substituted PAHs, while data on the bioactivation of alkyl-substituted PAHs are scarce. The present study investigated the effect of alkyl substitution on the CYP 450-mediated metabolism of phenanthrene and eight of its alkylated congeners by quantifying metabolite formation in rat and human liver microsomal incubations. Furthermore, the mutagenicity of four selected methylated phenanthrenes was compared to that of phenanthrene using the Ames test. The obtained results support the hypothesis that alkyl substitution shifts the oxidative metabolism from the aromatic ring to the alkyl side chain. Increasing the length of the alkyl chain reduced overall metabolism with metabolic conversion for 1-n-dodecyl-phenanthrene (C12) being negligible. 1- and 9-methyl-phenanthrene, in which the methyl group generates an additional bay region-like structural motif, showed mutagenicity toward Salmonella typhimurium TA98 and TA 100, whereas phenanthrene and also 2- and 3-methyl-phenanthrene, without such an additional bay region-like structural motif, tested negative. It is concluded that the position of the alkylation affects the metabolism and resulting mutagenicity of phenanthrene with the mutagenicity increasing in cases where the alkyl substituent creates an additional bay region-like structural motif, in spite of the extra possibilities for side chain oxidation.
\end{abstract}

Keywords Alkylated phenanthrene $\cdot$ Michaelis-Menten kinetics $\cdot$ Rat $\cdot$ Human $\cdot$ Microsomes $\cdot$ Mutagenicity

\section{Introduction}

The unintentional consumption of polycyclic aromatic hydrocarbons (PAHs) via contaminated food is a general food safety issue. The PAHs present in food are typically unsubstituted as they are pyrogenic in nature, formed by incomplete heating or combustion of organic matter (EFSA 2008). However, contamination of food with petrogenic polycyclic aromatic hydrocarbons (PPAHs), which are typically alkylated, is an emerging health concern (EFSA 2012; Fengler and Gruber 2020; Grob 2018; Pirow et al. 2019; Van Heyst et al. 2018). In spite of the fact that petroleum-derived mineral oils are highly refined to eliminate

Danlei Wang

danlei.wang@wur.nl

1 Division of Toxicology, Wageningen University and Research, 6708 WE Wageningen, The Netherlands undesirable substances such as mutagenic PAHs to make them compliant to EU regulations that forbid selling of carcinogenic substances to the general public (Carrillo et al. 2019), concerns about potentially carcinogenic constituents have been raised. It is known that some non-substituted and methylated polycyclic aromatic hydrocarbons with three to seven fused rings are mutagenic and potentially carcinogenic (Carrillo et al. 2019). Consumer products may be contaminated with PPAHs as a result of inappropriate use of mineral oils or via environmental contamination. For instance, crude oil spills, such as the Deepwater Horizon oil spill in the Gulf of Mexico, released large amounts of PPAHs into the environment (Fernando et al. 2019). In the Deepwater horizon oil spill phenanthrene and its methylated congeners were found to be among the most abundant PPAHs (NIST 2012). Part of these PPAHs may be taken up by marine species and end up in the food chain via consumption of seafood which has raised concerns for human health (Pulster et al. 


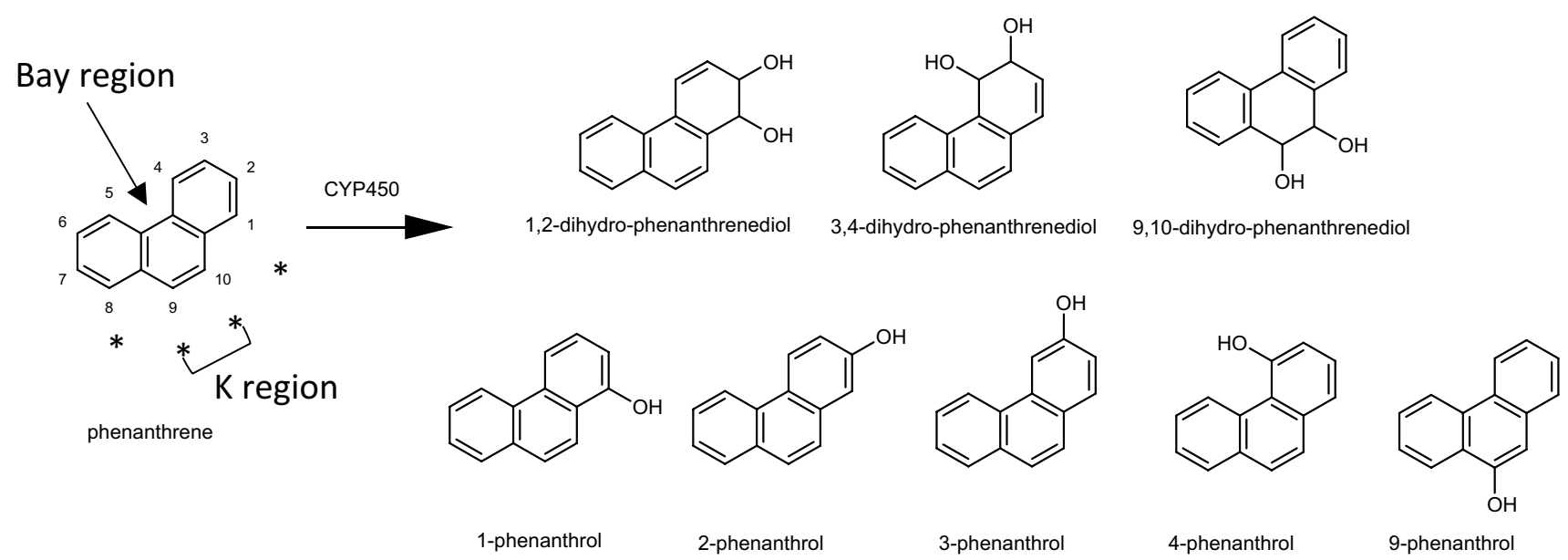

Fig. 1 Reported metabolites of phenanthrene formed in microsomal incubations by P450 enzymes of humans and rodents (Bao and Yang 1991; Chaturapit and Holder 1978; Jacob et al. 1996; Schober et al. 2010; Shou et al. 1994). *Indicates a peri position

2020; Ylitalo et al. 2012). The current knowledge on the possible metabolic activation and genotoxicity of PAHs primarily relates to unsubstituted PAHs, which are typically of pyrogenic origin. However, the potential bioactivation due to oxidative metabolism of substituted PAHs, such as PPAHs, has not been systematically investigated.

Some unsubstituted PAHs, such as benzo[a]pyrene and dibenzo[a,1]pyrene, are bioactivated by oxidation at the "bay region" or "fjord region" to highly reactive and mutagenic dihydrodiol-epoxides which may form adducts to DNA and eventually induce mutations and cancer (Boogaard 2012; Lehr et al. 1985; Tsang and Griffin 1979; Whalen et al. 1978). Information on the metabolism of PPAHs and their potential bioactivation is limited.

In our previous study, the in vitro hepatic biotransformation of naphthalene and alkyl-substituted naphthalenes was quantified (Wang et al. 2020). It was found that alkyl substitution of naphthalene shifts metabolism toward alkyl side chain oxidation at the cost of aromatic ring oxidation. To get more insight into the metabolic transformation of alkyl-substituted PAHs that may be present in mineral oils and crude oils, in the present study, the oxidative metabolism of phenanthrene and a series of its alkylated congeners is investigated.

Phenanthrene, the smallest PAH with a bay region, was classified by IARC as group 3 (not classifiable as to its carcinogenicity to humans), based on inadequate data in experimental animals (IARC, 2010). Phenanthrene can be metabolized by cytochrome $\mathrm{P} 450$ enzymes from humans or rodents to 1,2-dihydrodiol-, 3,4-dihydrodioland 9,10-dihydrodiol-phenanthrene, and 1-, 2-, 3-, 4- and 9-phenanthrols (Fig. 1) (Bao and Yang 1991; Chaturapit and Holder 1978; Jacob et al. 1996; Schober et al. 2010; Shou et al. 1994). However, no phenanthrene DNA adducts could be detected in Chinese hamster bone marrow cells following in vivo exposure to phenanthrene (Bayer 1978). Limited data on the metabolism of alkylated congeners of phenanthrene are available. Side chain hydroxylation was reported as the major pathway of 1-methylphenanthrene and 9-ethylphenanthrene in human HepG2 cells (Huang et al. 2017). Dihydrodiols of 1-hydroxymethylphenanthrene, dihydrodiols of 1-methyl-phenanthrene, 1-hydroxymethyl-phenanthrene and 1-methylphenananthrenols were found in incubations with rat $\mathrm{S} 9$ fractions and 1-methylphenanthrene (LaVoie et al. 1981). A similar metabolite profile was reported for 9-methylphenanthrene (LaVoie et al. 1981). Metabolic patterns and potential preferences for alkyl side chain oxidation or aromatic ring oxidation of other alkylated phenanthrenes, especially the ones with longer alkyl chains, are still unclear.

The model compounds included in the present study were phenanthrene, 1-methylphenanthrene, 2-methylphenanthrene, 3-methylphenanthrene, 9-methylphenanthrene, 2-ethylphenanthene, 9-ethylphenanthrene, 10-methyl-9-ethylphenanthrene, 1-n-hexylphenanthrene and 1-n-dodecylphenanthrene. The metabolic profile, kinetics and catalytic efficiency of the conversion of these model compounds were characterized in reactions likely mediated by cytochrome P450 in rat or human liver microsomes to better define the metabolic consequences of alkylation. In addition, some selected methylated congeners as well as unsubstituted phenanthrene were tested for their mutagenicity in the Ames test, to obtain further insight into the effect of the introduction of an additional bay region-like structural motif as a result of alkylation on the mutagenicity. 


\section{Material and methods}

\section{Chemicals and reagents}

Phenanthrene ( $\geq 98 \%)$, 2-ethylphenanthrene ( $\geq 98 \%)$, 9-ethylphenanthrene ( $\geq 98 \%), 10$-methyl-9-ethylphenanthrene ( $\geq 98 \%)$, 3-(hydroxymethyl)-phenanthrene ( $\geq 95.0 \%)$, 9-(hydroxymethyl)-phenanthrene ( $\geq 95.0 \%)$, tetrahydrofuran $(\geq 99.9 \%)$, trifluoroacetic acid ( $\geq 99 \%)$, methylmethanesulfonate, 2-aminoanthracene, and nitrofluorene were purchased from Sigma-Aldrich (St.Louis, USA). 1-Methylphenanthrene $(\geq 96 \%)$ was obtained from Toronto Research Chemicals (North York, Canada). 3-Methylphenanthrene ( $\geq 98 \%)$, 1-phenanthrol ( $\geq 98 \%)$, 3-phenanthrol ( $\geq 98 \%)$, 4-phenanthrol ( $\geq 98 \%$ ) and NADPH were supplied by Carbosynth (Berkshire, UK). 9-Methylphenanthrene ( $\geq 98 \%)$ and 2-methylphenanthrene $(\geq 98 \%)$ were purchased from BOC Sciences (Hamburg, Germany). 1-n-Hexylphenanthrene ( $\geq 99.2 \%)$ and 1-n-dodecylphenanthrene ( $\geq 99.5 \%)$ were synthesized by the Biochemical Institute for Environmental Carcinogens (Großhansdorf, Germany). Acetonitrile was bought from Biosolve (Dieuze, France). Dimethyl sulfoxide (DMSO), $\mathrm{K}_{2} \mathrm{HPO}_{4} \cdot 3 \mathrm{H}_{2} \mathrm{O}, \mathrm{MgCl}_{2}$, and $\mathrm{KCl}$ were supplied by Merck (Darmstadt, Germany). Gentest ${ }^{\mathrm{TM}}$ pooled male Sprague-Dawley rat liver microsomes (RLM) and Ultrapool $^{\mathrm{TM}}$ human liver microsomes (HLM) with a protein concentration of $20 \mathrm{mg} / \mathrm{ml}$ were obtained from Corning (New York, USA), and the latter contained cytochrome P450 liver enzymes of 150 individuals. Rat liver S9 homogenate was obtained from Trinova Biochem $\mathrm{GmbH}$ (Giessen, Germany) and was prepared from the livers of male Sprague-Dawley rats that had been injected intraperitoneally with Aroclor 1254 (500 mg/kg body weight). Salmonella typhimurium TA98 and TA 100 tester strains were also obtained from Trinova Biochem GmbH (Giessen, Germany). NADP and glucose-6-phosphate were supplied by Randox Laboratories Ltd. (Crumlin, UK) and Roche Diagnostics (Mannheim, Germany), respectively.

\section{In vitro incubations of phenanthrene and its alkylated congeners with rat and human liver microsomes}

Microsomal oxidation of phenanthrene and its alkylated congeners by RLM and HLM was investigated in an overall $200 \mu \mathrm{l}$ incubation system consisting of potassium phosphate $(0.1 \mathrm{M}, \mathrm{pH} 7.4)$, containing $5 \mathrm{mM} \mathrm{MgCl}_{2}$, RLM/HLM at a final microsomal protein concentration of $0.5 \mathrm{mg} / \mathrm{ml}, 1 \mathrm{mM} \mathrm{NADPH}$, and each of the individual test compounds at concentrations ranging from 0 to $600 \mu \mathrm{M}$. Test compounds were phenanthrene, 1-methylphenanthrene, 2-methylphenanthrene, 3-methylphenanthrene, 9-methylphenanthrene, 2-ethylphenanthrene, 9-ethylphenanthrene, 10-methyl-9-ethylphenanthrene, 1-n-hexylphenanthrene and 1-n-dodecyl-phenanthrene. The final concentration of substrate solvent, either DMSO or tetrahydrofuran (the latter was used for 1-n-hexylphenanthrene and 1-n-dodecylphenanthrene due to their low solubility in DMSO) in the incubation mixture was $1 \%$ $(v / v)$, which did not affect the enzymatic activity of liver microsomes ( $\mathrm{Li}$ et al. 2010). The incubation mixtures were prepared and incubated in glass vials to avoid plastic binding of the substrates. The glass vials were capped to prevent substrate loss due to volatility. After preincubation of the incubation mixture at $37{ }^{\circ} \mathrm{C}$ for $1 \mathrm{~min}$, the enzymatic reaction was initiated by adding microsomes to the incubation mixture which was subsequently incubated at $37{ }^{\circ} \mathrm{C}$ for $20 \mathrm{~min}$. The reaction was terminated by adding $100 \mu \mathrm{l}$ ice-cold acetonitrile followed by vortexing. After $5 \mathrm{~min}$ centrifugation at $5000 \mathrm{rpm}(4000 \times g), 4{ }^{\circ} \mathrm{C}$, the supernatant was collected for ultraperformance liquid chromatography (UPLC) analysis. However, the concentrations of the metabolites of two of the test substrates, 1-n-hexylphenanthrene and 1-n-dodecyl-phenanthrene, in the supernatant appeared too low to detect metabolism. Therefore, a diisopropylether (DIPE) extraction of the metabolites was performed after the reaction was stopped by the addition of $20 \mu \mathrm{l} 10 \% \mathrm{HClO}_{4}$. To this end, the incubation mixture (total volume of $220 \mu \mathrm{l}$ ) was extracted three times with $1 \mathrm{ml}$ DIPE. Each time, the upper organic layer was collected and the DIPE was subsequently evaporated from the combined organic fractions with a gentle stream of nitrogen. The residues were dissolved in $100 \mu$ methanol and analyzed by UPLC.

To prepare samples for metabolite identification by GC-MS/MS, each individual test substance $(200 \mu \mathrm{M}$ final concentration) was incubated in a volume of $400 \mu \mathrm{l}$ in potassium phosphate $(0.1 \mathrm{M}, \mathrm{pH}$ 7.4) containing $5 \mathrm{mM}$ $\mathrm{MgCl}_{2}, \mathrm{RLM}$ or HLM at a final microsomal protein concentration of $1 \mathrm{mg} / \mathrm{ml}$, and $1 \mathrm{mM} \mathrm{NADPH}$ at $37^{\circ} \mathrm{C}$ for $10 \mathrm{~min}$. After incubation, the incubation mixture was centrifuged for $5 \mathrm{~min}$ at $5000 \mathrm{rpm}(4000 \times \mathrm{g})$ and $4{ }^{\circ} \mathrm{C}$. The supernatant of the incubation mixture was transferred to a fresh vial and extracted three times with $100 \mu \mathrm{l}$ dichloromethane (DCM) following vortexing. The DCM phase containing the substrate and metabolites was separated from the aqueous phase by centrifugation at $5000 \mathrm{rpm}$ $(4000 \times g)$ for $5 \mathrm{~min}$. The organic (lower) phase was collected from each extraction, combined and analyzed by GC-MS/MS. 


\section{Reverse mutation assay}

The mutagenicity of phenanthrene and four of its methylated analogs (1-methyl-, 2-methyl-, 3-methyl- and 9-methylphenanthrene) was assessed in the Ames test, a bacterial reverse mutation assay, using the TA98 and TA100 strains of S. typhimurium. Six concentrations of each compound were tested in triplicate in the absence and presence of 5\% $(\mathrm{v} / \mathrm{v})$ S9-mix prepared from the livers of Aroclor 1254 treated Sprague-Dawley rats. The S9-mix contained 4 mM NADP, $5.8 \mathrm{mM}$ glucose-6-phosphate, $0.1 \mathrm{M}$ sodium phosphate $\mathrm{pH}$ 7.4, $8 \mathrm{mM} \mathrm{MgCl}_{2}, 33 \mathrm{mM} \mathrm{KCl}$ and $5 \% \mathrm{~S} 9$ homogenate. Fresh bacterial cultures were prepared overnight to reach $10^{9} \mathrm{cells} / \mathrm{ml}$. The following solutions were pre-incubated in a rotating incubator at $70 \mathrm{rpm}$ and $37^{\circ} \mathrm{C}$, and contained either $0.5 \mathrm{ml} \mathrm{S} 9-\operatorname{mix}$ (in case of $\mathrm{S} 9$ presence) or $0.5 \mathrm{ml} 0.1 \mathrm{M}$ potassium phosphate $\mathrm{pH} 7.4$ (in case of $\mathrm{S} 9$ absence), $0.1 \mathrm{ml}$ of a fresh bacterial culture $\left(10^{9} \mathrm{cells} / \mathrm{ml}\right)$ of TA98 or TA100, and 10-100 $\mu \mathrm{g}$ test compound. Top agar was melted and heated to $45^{\circ} \mathrm{C}$. After preincubation, the substrate solutions were added to $3 \mathrm{ml}$ of the molten top agar and mixed by vortexing and the top agar mixture was poured onto a minimal glucose agar plate. After solidification of the top agar, the plates were incubated at $37^{\circ} \mathrm{C}$ for $48 \mathrm{~h}$. The number of revertant colonies per plate was automatically counted with the Instem Sorcerer Colony Counter (Staffordshire, UK). In the absence of S9-mix, 2-nitrofluorene (NF) and methylmethanesulfonate (MMS) were tested as positive controls for incubations with TA98 and TA100, respectively. In the presence of S9-mix, 2-aminoanthracene (2AA) was tested as a positive control in both TA98 and TA100. DMSO was tested as a solvent control in both tester strains. The mutagenicity of the test compounds was determined by the number of revertant colonies per plate and considered positive if the revertant number was increased compared to the historical control data and was also more than threefold or twofold higher than the controls for tester strain TA98 and TA100, respectively (Levy et al. 2019). The historical control data are presented in Table $\mathrm{S} 1$ in the supplementary material 1.

\section{In vitro incubations of phenanthrene and its methylated analogs with rat S9}

Since the reverse mutation assay used an S9 metabolic system instead of microsomes, for the compounds tested in the reverse mutation assay the metabolite patterns were also characterized in incubations with rat liver S9. To this end, the incubation mixture of each compound tested in the reverse mutation assay with Aroclor 1254-treated rat liver S9 was analyzed by UPLC, while unidentified metabolites were further analyzed by GC-MS/MS. Incubation mixtures consisting of $100 \mu \mathrm{g}(1000 \mu \mathrm{M}$ final concentration) test compound, either $0.5 \mathrm{ml} \mathrm{S9-mix} \mathrm{(in} \mathrm{case} \mathrm{of} \mathrm{S9} \mathrm{presence)}$ or $0.5 \mathrm{ml} 0.1 \mathrm{M}$ potassium phosphate $\mathrm{pH} 7.4$ (in case of $\mathrm{S} 9$ absence) were incubated for $48 \mathrm{~h}$ at $37^{\circ} \mathrm{C}$, applying the concentrations, incubation time and temperature also used in the Ames assay. After the 48-h incubation, $250 \mu \mathrm{l}$ acetonitrile was added to the incubation mixture, followed by centrifugation at $5000 \mathrm{rpm}(4000 \times \mathrm{g})$ for $5 \mathrm{~min}$. The supernatant was collected for UPLC analysis. For GC-MS/MS analysis, the incubation mixture was centrifuged for $5 \mathrm{~min}$ at $5000 \mathrm{rpm}(4000 \times g)$ and $4{ }^{\circ} \mathrm{C}$ after the 48 -h incubation. The supernatant thus obtained was transferred to a fresh vial and extracted three times with $100 \mu \mathrm{l}$ DCM following vortexing. The DCM phase, containing the substrate and its metabolites, was separated from the aqueous phase by centrifugation at $4000 \times g$ for $5 \mathrm{~min}$. The organic (lower) phase was collected, combined and analyzed by GC-MS/MS.

\section{UPLC analysis}

The metabolites formed were analyzed and quantified using an Acquity UPLC system equipped with a photodiode array (PDA) detector (Waters, Milford, MA). The metabolites and their parent compound were separated on a reverse phase Acquity UPLC ${ }^{\circledR}$ BEH C 18 column $(21 \times 50 \mathrm{~mm}, 1.7 \mu \mathrm{m}$, Waters, Milford, MA) and detected at wavelengths ranging from 190 to $400 \mathrm{~nm}$. Eluent A was nano-pure water containing $0.1 \%$ trifluoroacetic acid $(v / v)$, and eluent B was acetonitrile containing $0.1 \%$ trifluoroacetic acid $(v / v)$. The gradient elution started from $90 \% \mathrm{~A}$ and $10 \% \mathrm{~B}$ applied from $0.0 \mathrm{~min}$ to $0.5 \mathrm{~min}$, which was changed to $0 \% \mathrm{~A}$ and $100 \% \mathrm{~B}$ from 0.5 to $15.5 \mathrm{~min}$ and then kept at $0 \% \mathrm{~A}$ and $100 \% \mathrm{~B}$ from 15.5 min to 18.5 min, changed back to $90 \% \mathrm{~A}$ and $10 \% \mathrm{~B}$ from 18.5 to $18.6 \mathrm{~min}$ and then maintained at the starting conditions from $18.6 \mathrm{~min}$ until $22 \mathrm{~min}$. The total run time was $22 \mathrm{~min}$ using a flow rate of $0.6 \mathrm{ml} / \mathrm{min}$. The temperature of the column was set at $40{ }^{\circ} \mathrm{C}$ and that of the autosampler at $10{ }^{\circ} \mathrm{C}$ during the UPLC analysis. The injection volume was $3.5 \mu \mathrm{l}$. Metabolites were quantified using their peak area at the wavelength specified in Table 1, using calibration curves of available reference compounds. Metabolites were identified by comparing their retention time (RT) and UV spectrum to those of reference chemicals on UPLC. When reference chemicals were not available, metabolite identification by gas chromatography-triple quadrupole mass spectrometry (GC-MS/MS) was performed. The minor metabolites were identified by elution time and mass spectra both on UPLC and GC-MS/MS, and by comparison with available elution and spectral information from the literature.

Under the conditions used metabolite formation was linear with time and the amount of microsomal protein. The metabolite concentrations in the microsomal incubation mixtures as quantified by UPLC were used to calculate the rate of the enzymatic conversions in $\mathrm{pmol} / \mathrm{min} / \mathrm{mg}$ microsomal protein. The kinetic parameters $K_{M}$ and $V_{\max }$ were obtained 


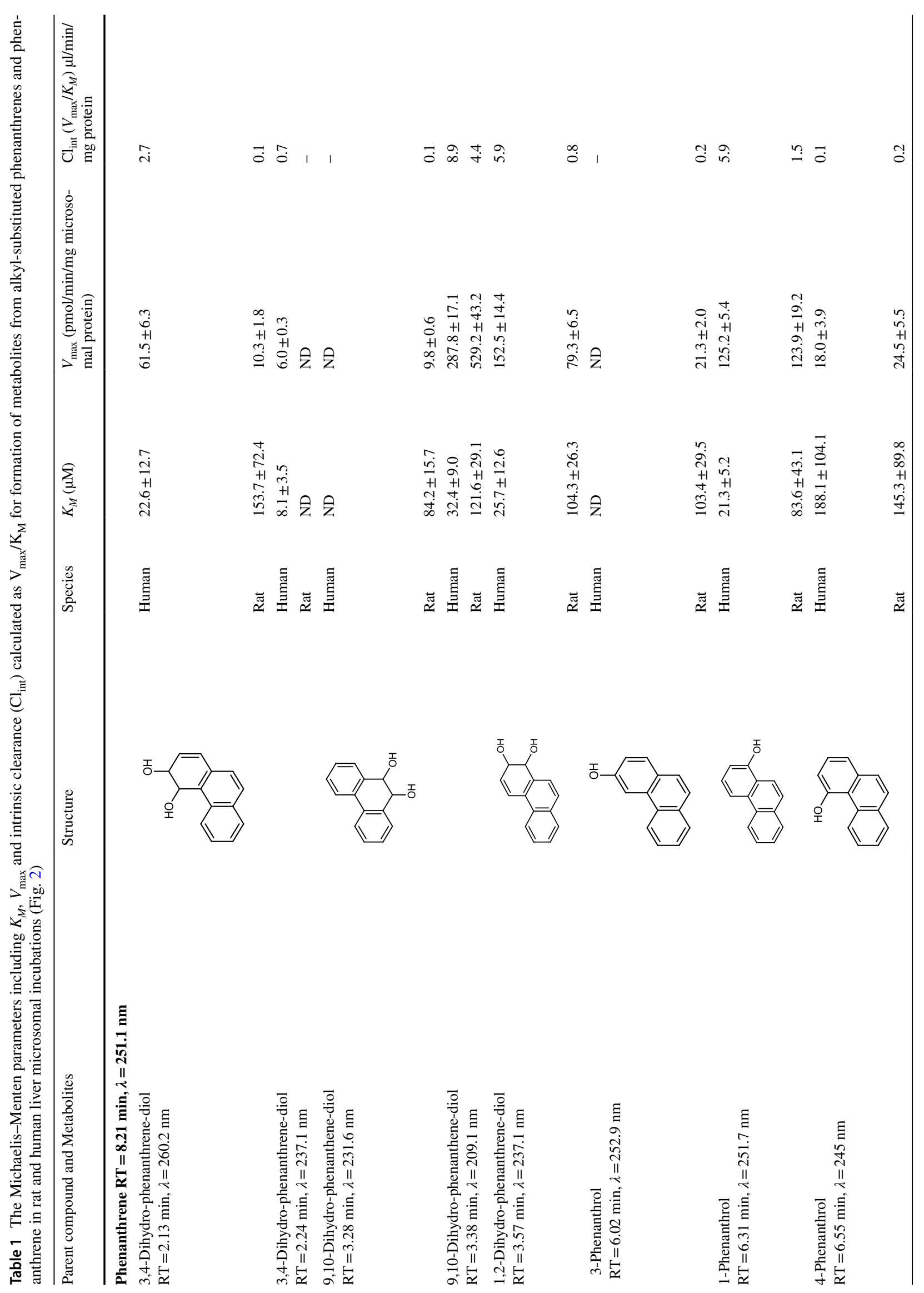




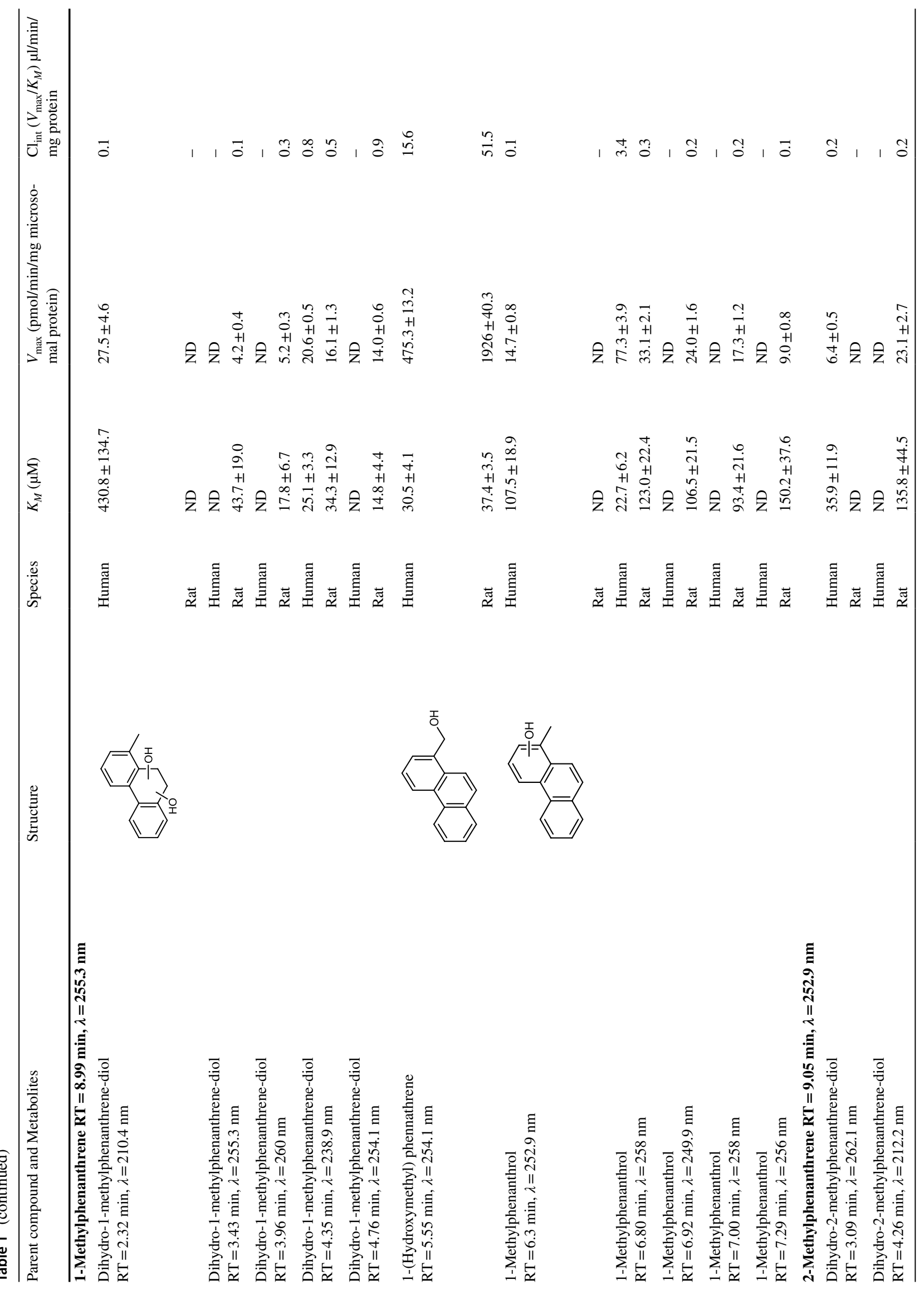




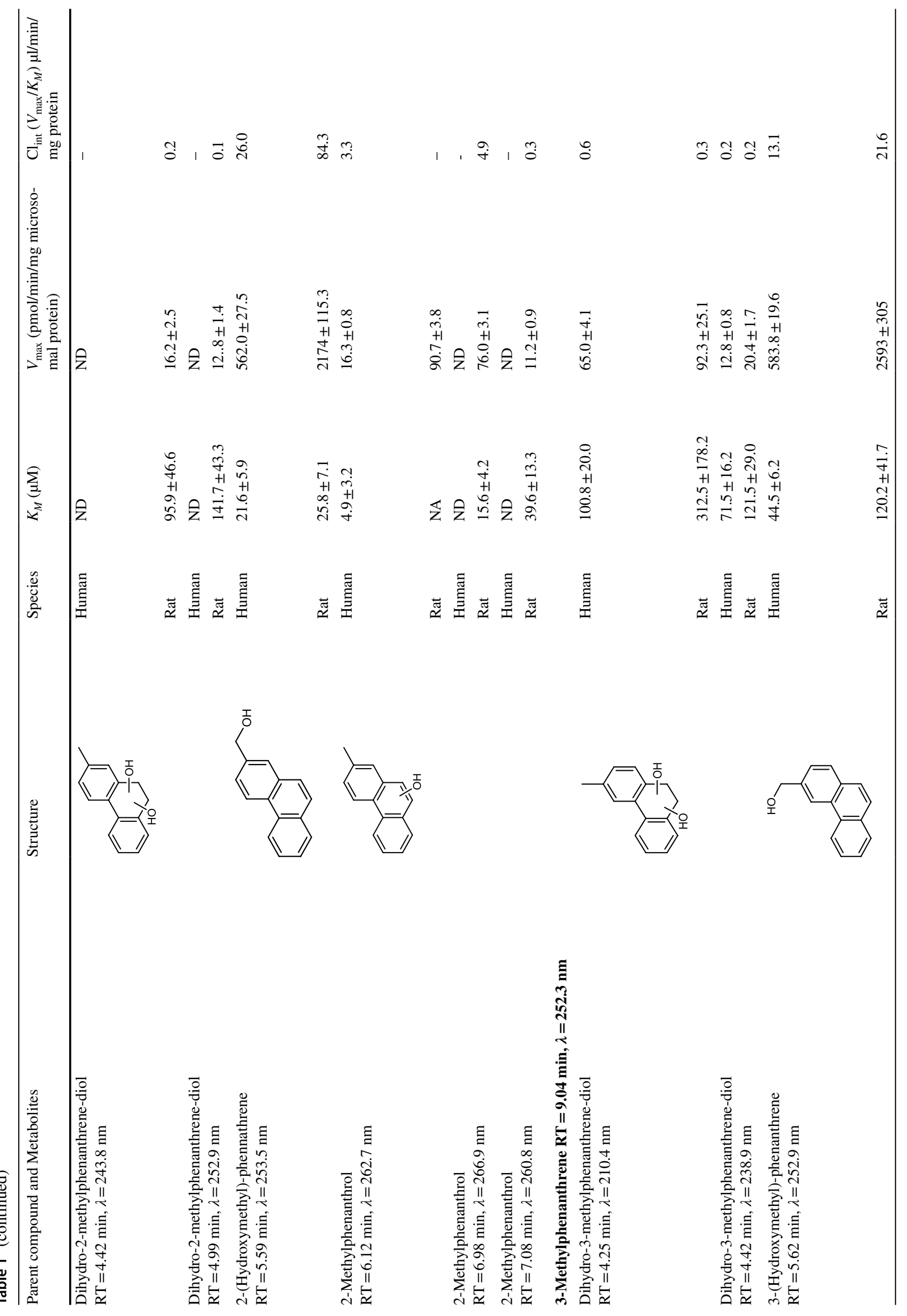




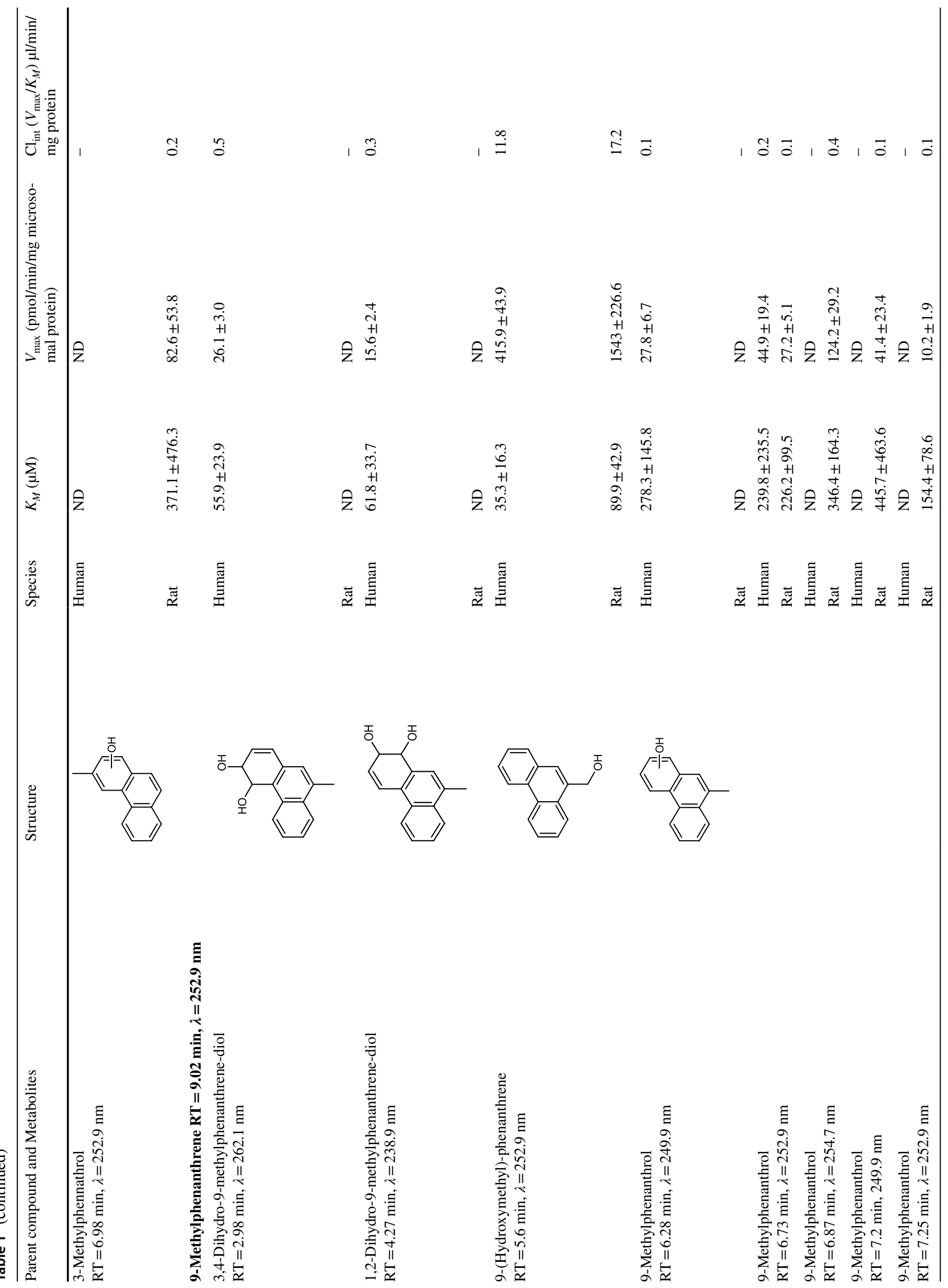




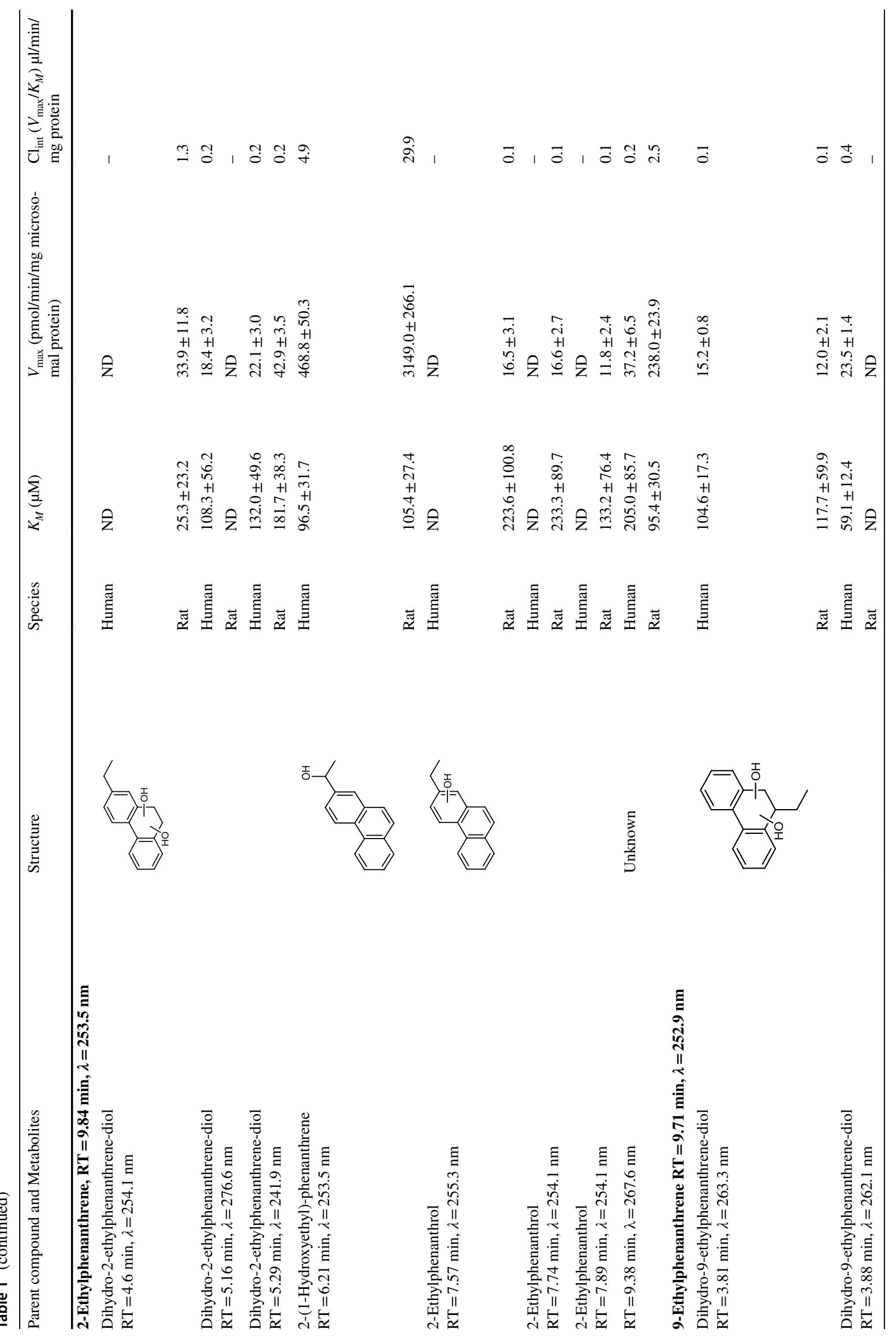


1118

Archives of Toxicology (2022) 96:1109-1131

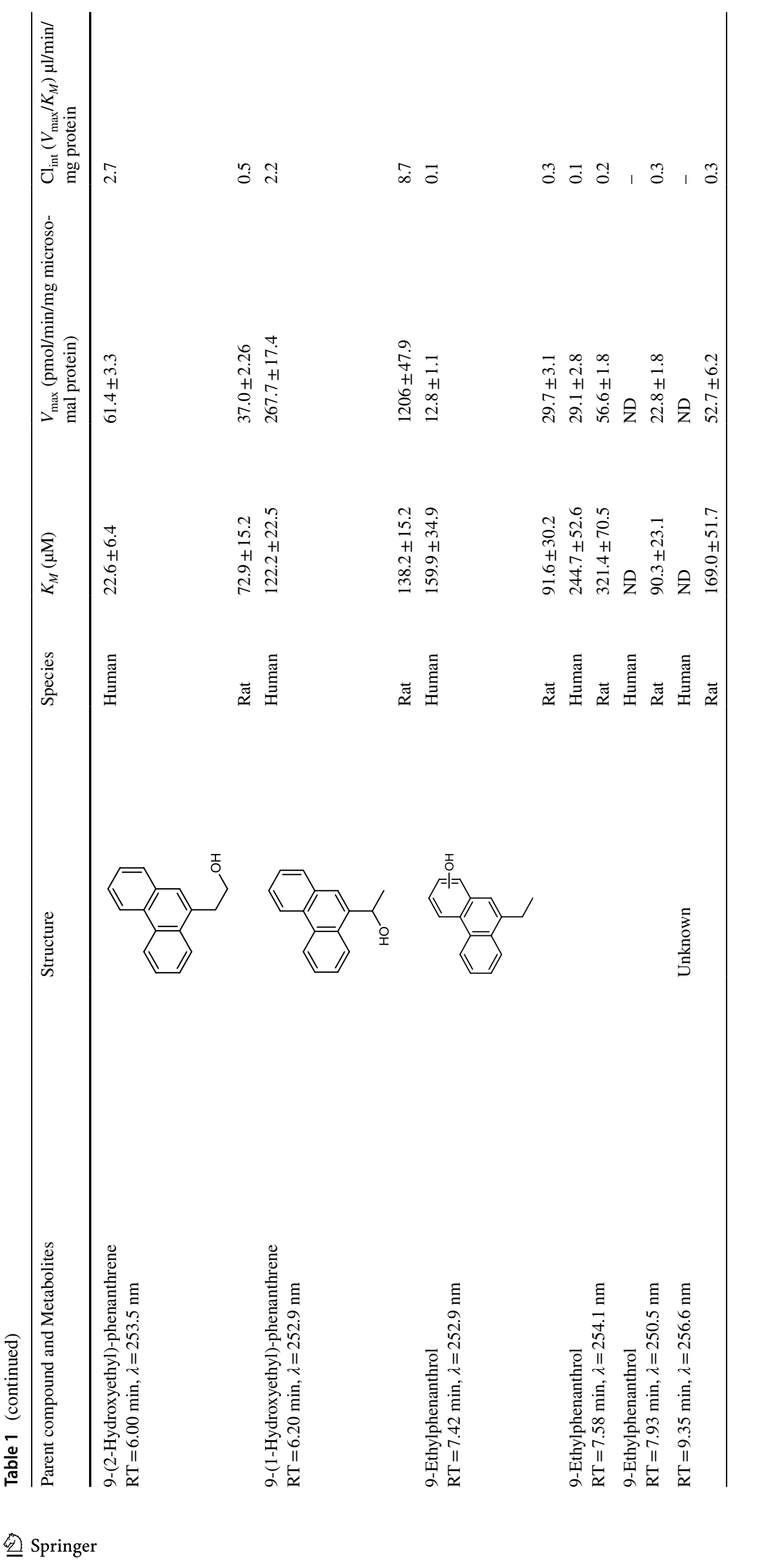




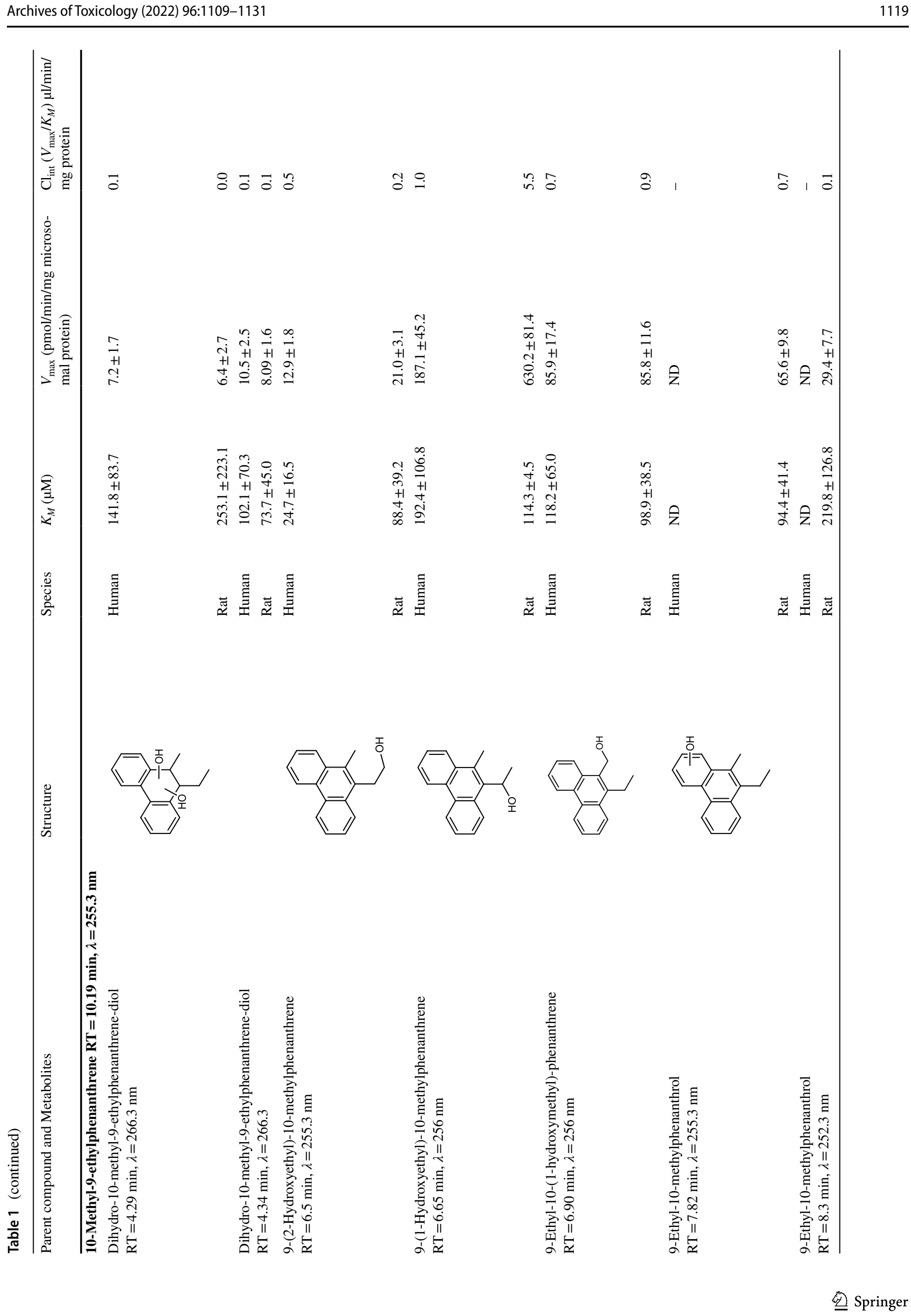




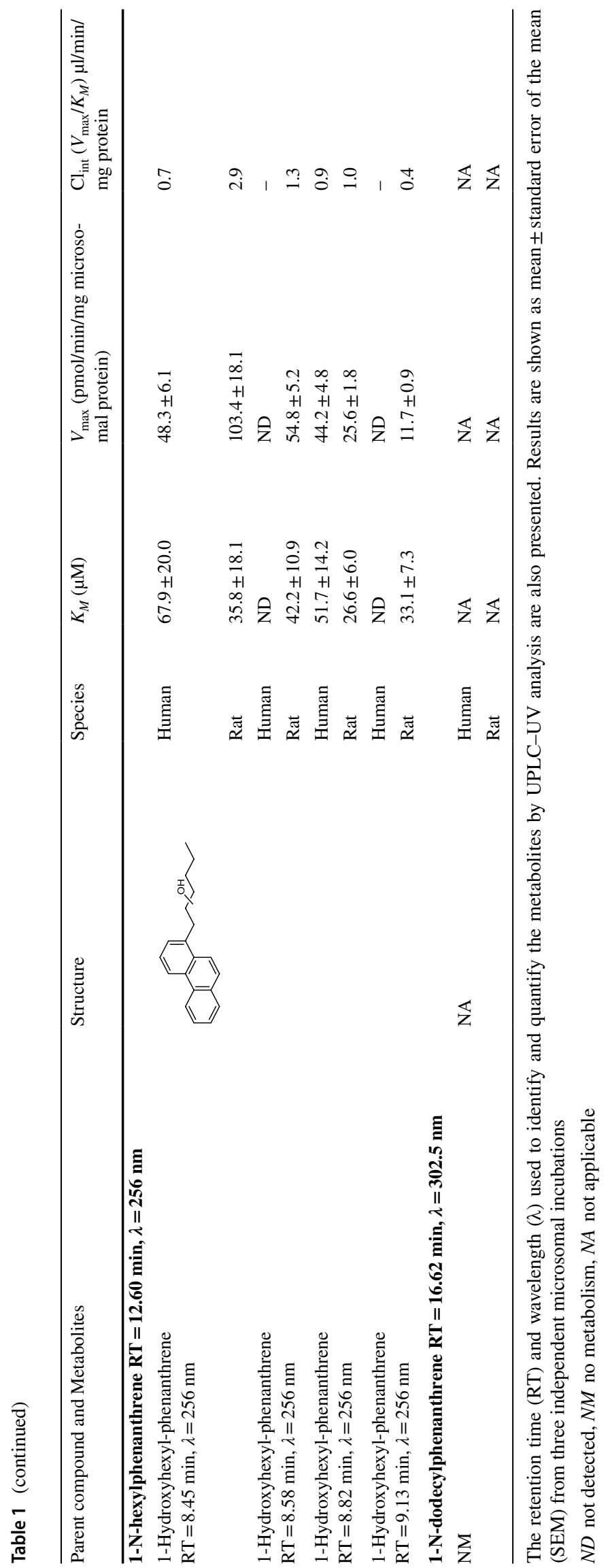


Fig. 2 Substrate concentrationdependent metabolism of alkyl-substituted phenanthrenes and phenanthrene itself by RLM and HLM. Green lines represent metabolite formation via alkyl chain oxidation and black lines present metabolite formation by aromatic ring oxidation. Each symbol represents experimental means and vertical bars are standard errors of the mean $(n=3)$ (color figure online)
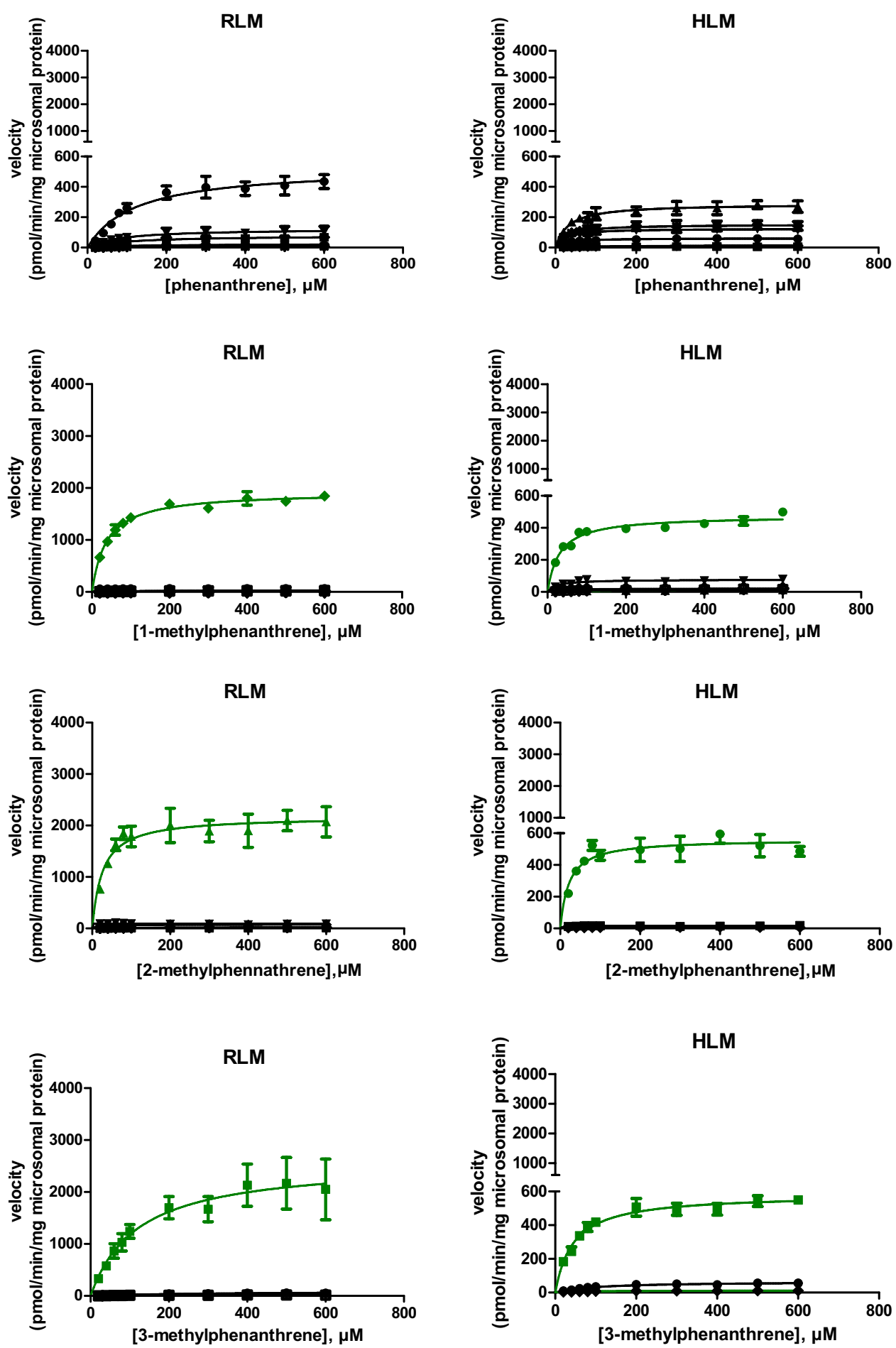

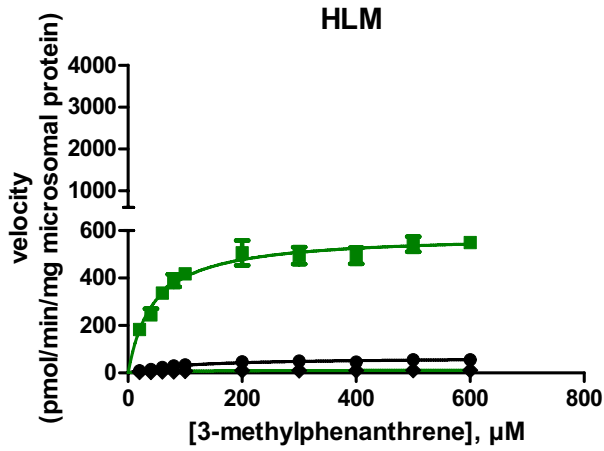

using a nonlinear regression curve fit applying the Michaelis Menten equation in GraphPad Prism 5 (San Diego, USA). To compare the catalytic efficiency of formation of the different metabolites the intrinsic clearance $\left(\mathrm{Cl}_{\text {int }}\right)$ was calculated as $V_{\max }$ divided by $K_{M}$.

\section{Metabolite identification by GC-MS/MS}

The MS spectra of all metabolites were recorded and compared to mass spectra from the NIST library (14, $14 \mathrm{~s}, 17-1$, 17-2, 17 s) available in the GC-MS/MS solution software Version 4.45 (Shimadzu, Japan). 
Fig. 2 (continued)
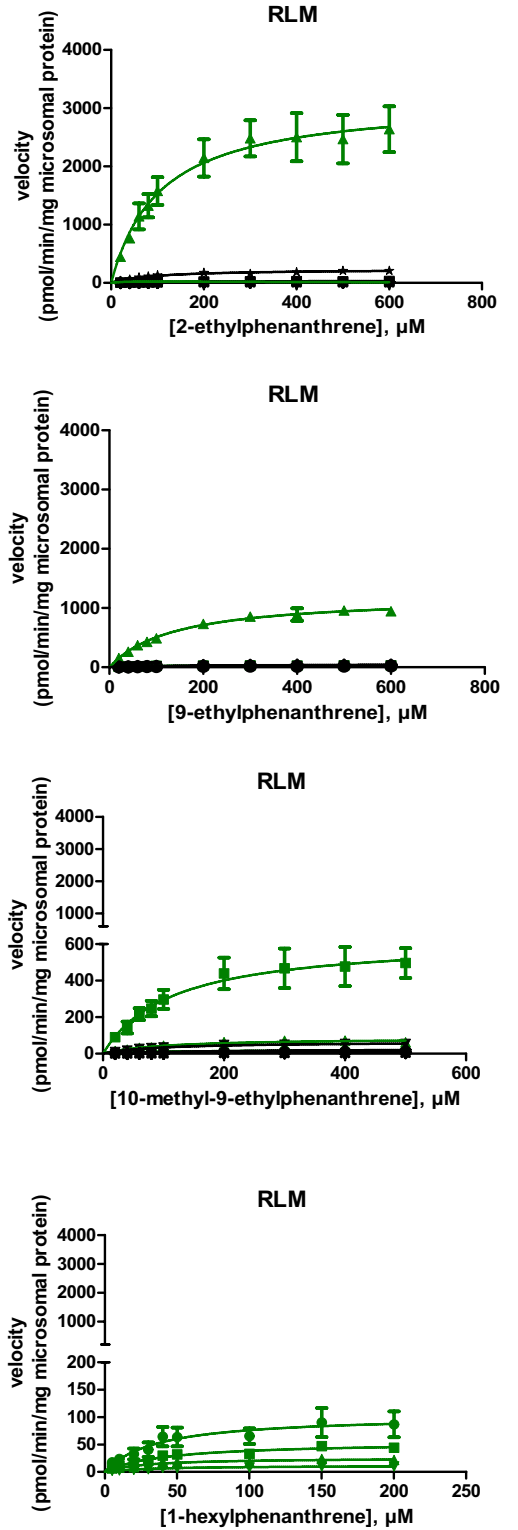

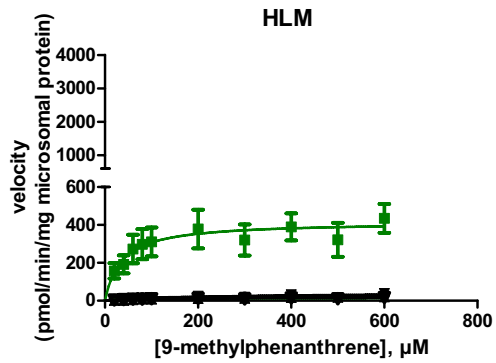

HLM
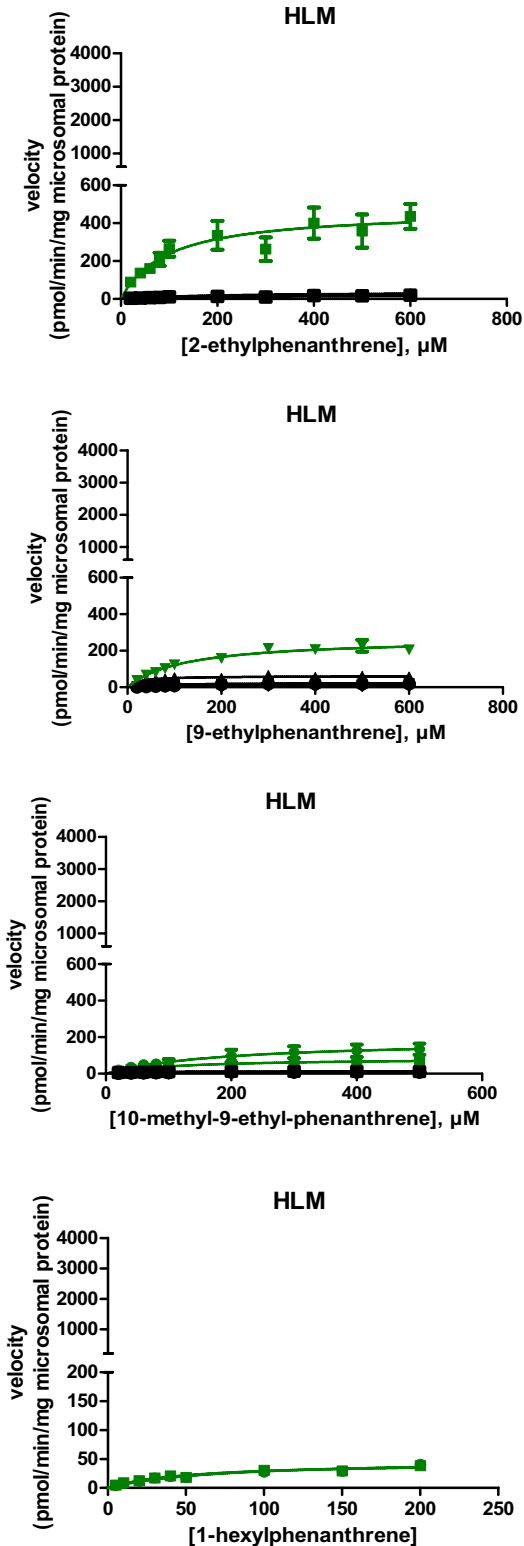

The metabolites formed from phenanthrene, 1-methylphenanthrene, 2-methylphenanthrene, 3-methylphenanthrene, 9-methylphenanthrene, 2-ethylphenanthrene, 9-ethylphenanthrene, 10-methyl-9-ethylphenanthrene, 1-n-hexylphenanthrene and 1-n-dodecylphenanthrene were analyzed using a Shimadzu GC-MS/MS system consisting of a GC-2010 Plus coupled with a mass spectrometer TQ8040 (Shimadzu, Japan). A $30 \mathrm{~m}$ capillary column with $0.25 \mathrm{~mm}$ diameter (ZB-1, Phenomenex, USA) was used to separate the metabolites upon injection of $1 \mu \mathrm{l}$ of the extract with splitless injection mode, using a constant flow of helium gas $(1 \mathrm{ml} / \mathrm{min})$. The column oven temperature 
started at $50{ }^{\circ} \mathrm{C}$ and 1 -min hold, increased to $300{ }^{\circ} \mathrm{C}$ at a rate of $20^{\circ} \mathrm{C} / \mathrm{min}$ from 1 to $13.5 \mathrm{~min}$, followed by $8.5 \mathrm{~min}$ hold at $300{ }^{\circ} \mathrm{C}$. The total run time was $22 \mathrm{~min}$ and electron ionization $(70 \mathrm{eV})$ was used to generate the ions of metabolites for mass spectrometric detection.

\section{Results}

\section{Microsomal metabolism of phenanthrene and its alkylated congeners}

The concentration-dependent rate of metabolite formation and the corresponding fitted curves representing Michaelis-Menten kinetics of the metabolite formation mediated by RLM and HLM are shown in Fig. 2 for the model compounds. The tested compounds were phenanthrene, 1-methylphenanthrene, 2-methylphenanthrene, 3-methylphenanthrene, 9-methylphenanthrene, 2-ethylphenanthrene, 9-ethylphenanthrene, 10-methyl-9-ethyl-phenanthrene and 1-n-hexylphenanthrene. No metabolic conversion was observed for 1-n-dodecyl-phenanthrene under the experimental conditions used. The obtained $K_{M}$ and $V_{\max }$ values and the calculated $\mathrm{Cl}_{\text {int }}$ for formation of each metabolite derived from the curves presented in Fig. 2 are summarized in Table 1.

Dihydro-phenanthrene-diols and phenanthrols were detected in both rat and human liver microsomal incubations of phenanthrene. Specifically, three major dihydro-phenanthrene-diols, 3,4-dihydro-phenanthrene-diol, 9,10-dihydrophenanthrene-diol, and 1,2-dihydro-phenanthrene-diol, were characterized based on comparison with reference

(a)

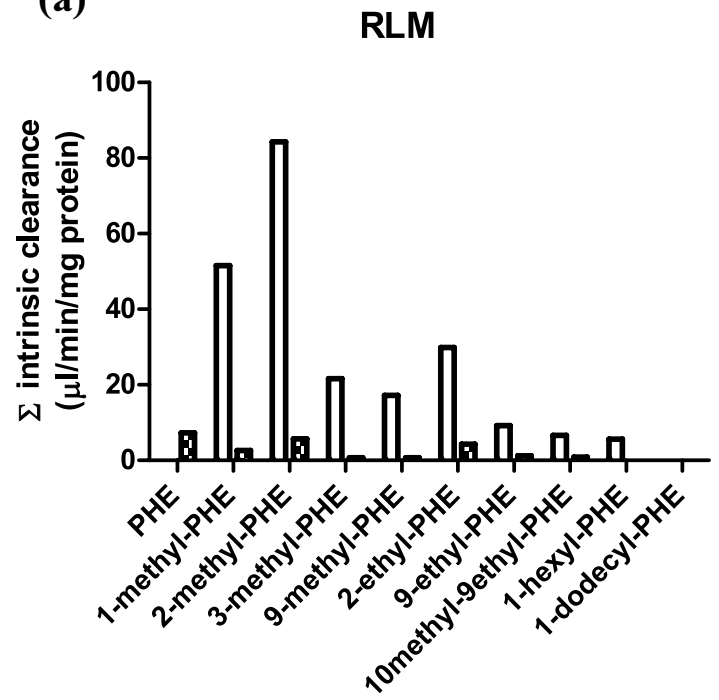

materials and the available literature (Bao and Yang 1991; Chaturapit and Holder 1978; Jacob et al. 1996; Schober et al. 2010; Shou et al. 1994; Sims 1970). For 3,4-dihydrophenanthrene-diol and 9,10-dihydro-phenanthrene-diol, two partially overlapping peaks, representing their respective cisand trans-isomers, were detected at 2.13 and $2.24 \mathrm{~min}$ and at 3.28 and $3.38 \mathrm{~min}$, respectively. Three phenanthrols were identified as 3-phenanthrol, 1-phenanthrol, and 4-phenanthrol by co-elution and identical UV spectra with the reference standards.

The oxidative metabolism of alkyl-substituted phenanthrenes primarily occurred on the alkyl chain. The most abundant type of metabolites detected in both rat and human liver microsomal incubation mixtures with the alkyl-substituted phenanthrenes were alcohols. The primary metabolites of 3-methylphenanthrene and 9-methylphenanthrene were identified as 3-hydroxymethyl-phenanthrene and 9-hydroxymethyl-phenanthrene, respectively, which co-eluted with the reference standards in both UPLC and GC-MS/MS analyses. The molecular ion and base peak of both 3-hydroxymethyl-phenanthrene and 9-hydroxymethyl-phenanthrene were observed at m/z 208 and m/z 179, respectively. A comparable mass spectrum was obtained for the primary metabolite of 1-methylphenanthrene with molecular ion at $\mathrm{m} / \mathrm{z} 208$ and a base peak at $\mathrm{m} / \mathrm{z} 179$ supporting its identification as 1-hydroxymethyl-phenanthrene with an identical UV spectra as reported for this compound (Huang et al. 2017). With a similar mass spectral profile and elution pattern, 2-hydroxymethyl-phenanthrene was identified as a primary metabolite of 2-methylphenanthrene. 2-(1-Hydroxyethyl)phenanthrene and 9-(1-hydroxyethyl)-phenanthrene were found to be the primary metabolites of 2-ethylphenanthrene (b)

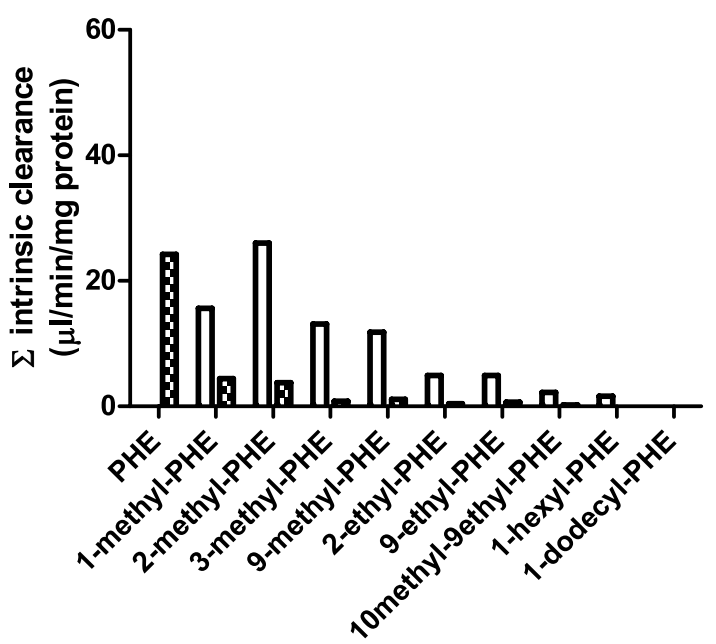

Fig. 3 Intrinsic clearance via aromatic ring and alkyl chain oxidation by a RLM and b HLM for the different model compounds. Dashed bars represent aromatic ring oxidation; white bars represent alkyl chain oxidation. Abbreviation. PHE = phenanthrene 
and 9-ethylphenanthrene, respectively, with a molecular ion at $m / z 222$ and a base peak at $m / z$ 179. In analogy with these results, the most abundant metabolite of 10-methyl-9-ethylphenanthrene was tentatively identified as 10-methyl-9-(1hydroxyethyl)-phenanthrene with a base peak at $\mathrm{m} / \mathrm{z} 203$, a molecular ion peak at $m / z 236$ and the presence of other ion peaks at $\mathrm{m} / \mathrm{z}, 179$ and $\mathrm{m} / \mathrm{z} 218$.

For alkylated phenanthrenes, dihydro-phenanthrene-diols and phenanthrols were minor metabolites formed in microsomal incubations with both RLM and HLM. On UPLC the most polar dihydrodiols eluted first, followed by alcohols, and subsequently by less polar phenanthrols before the parent compound eluted (Table 1), whereas on GC-MS/MS the parent compound with lowest boiling point eluted before the alcohols, dihydrodiols and phenanthrols. Identification of the minor metabolites was based on comparison with reference mass spectra in the NIST libraries or mass spectra of a reference compound that shared a similar structure. The detailed identification of the different microsomal metabolites formed from the alkyl-substituted phenanthrenes can be found in supplementary material 2 .

\section{Intrinsic clearance by aromatic and alkyl side chain oxidation}

To compare the metabolic efficiency of alkyl side chain oxidation and aromatic ring oxidation of phenanthrene and its alkyl-substituted analogs, the intrinsic clearance of each parent test compound via side chain metabolites and aromatic ring metabolites was calculated (Fig. 3). The overall intrinsic clearance of phenanthrene was 7.3 and $24.2 \mu \mathrm{l} / \mathrm{min} / \mathrm{mg}$ protein when metabolized by hepatic microsomes of rat and human, respectively. With increasing chain length the intrinsic clearance decreased substantially with for phenanthrenes with an alkyl side chain with more than three carbon atoms clearance being limited or even (1-n-dodecylphenanthrene) not observed at all (Fig. 3). The results presented in Fig. 3 also reveal that when phenanthrene was alkyl-substituted aromatic ring oxidation was reduced in favor of alkyl side chain oxidation. When there were more than 3 carbon atoms in the alkyl side chain of the tested substrates, aromatic ring oxidation was no longer detectable. In case of HLM, the intrinsic clearance of the tested alkylated phenanthrenes for which aromatic ring oxidation was observed was 5.5-121 times lower than that of phenanthrene. For metabolism by HLM of the tested substrates with up to three carbon atoms in the alkyl side chain, the intrinsic clearance via alkyl side chain oxidation was 3.5-16.4 times greater than that via aromatic ring oxidation. RLM metabolized the alkylated phenanthrenes via side chain oxidation 1.6-6.1 times more efficiently than HLM. For RLM the intrinsic clearance via aromatic ring oxidation of the tested alkylated phenanthrenes was 1.3- to 10.4-fold lower than that of phenanthrene itself. For the alkylated phenanthrenes for which aromatic oxidation was observed, the alkyl side chain oxidation was 7.0-30.8 times more efficient than the aromatic ring oxidation in microsomal incubations with RLM.

\section{Effect of alkyl chain length on total intrinsic clearance}

By adding up the intrinsic clearance of all metabolites for each substrate, the total intrinsic clearance of phenanthrene with and without alkyl substitution based on side chain carbon number was calculated. Figure 4 presents this overall intrinsic clearance via aromatic ring and side chain oxidation as a function of the number of carbon atoms in the alkyl side chain for the different model compounds. The results (a)

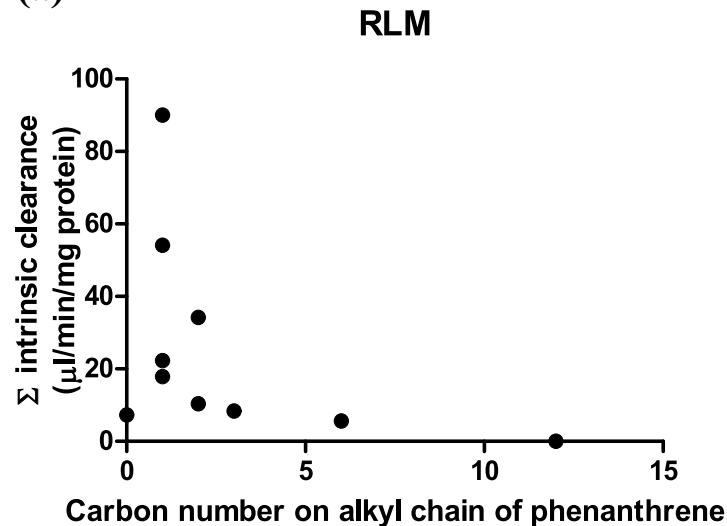

Fig. 4 Relationship between total $\mathrm{Cl}_{\text {int }}$ of alkylated phenanthrenes and the number of carbon atoms in the alkyl side chain in metabolism with a RLM and b HLM. The intrinsic clearance was calculated by (b)

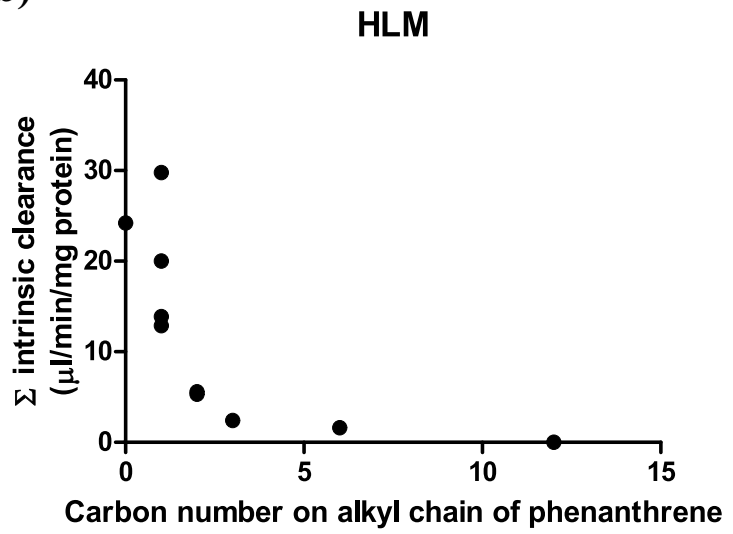

adding up the $\mathrm{Cl}_{\text {int }}$ values of all metabolites for the respective model compound (Table 1) 
(a)

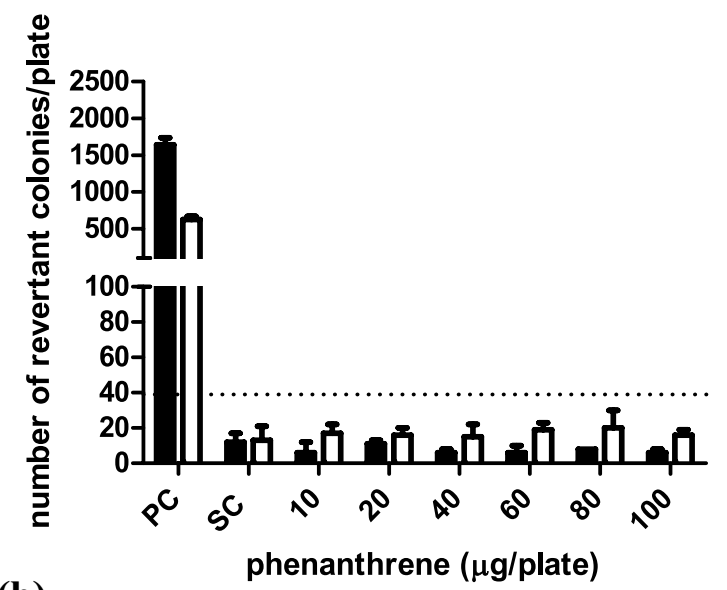

(b)

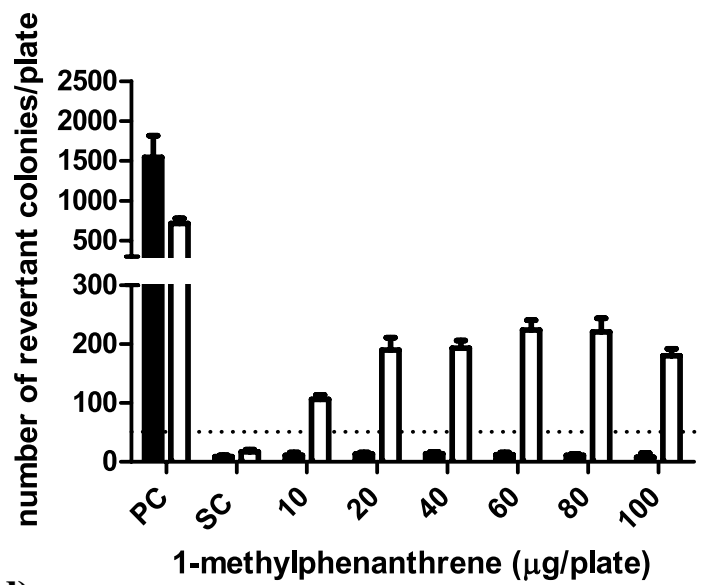

(d)

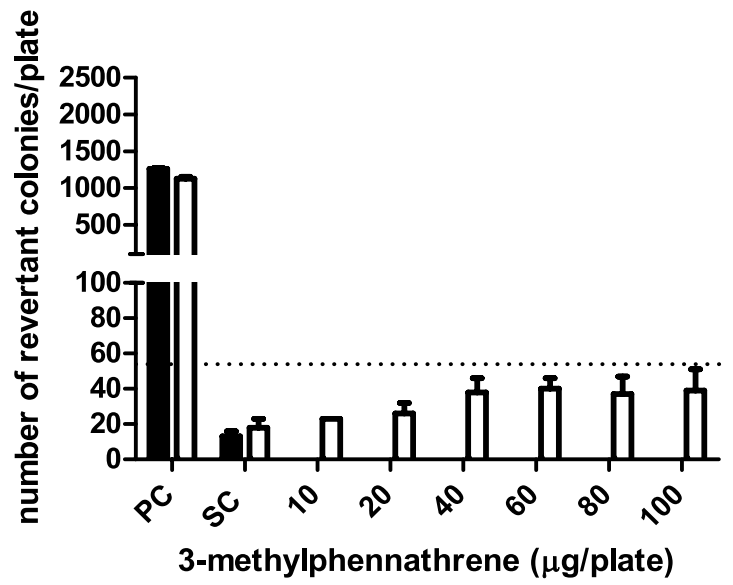

Fig. 5 Number of revertants in S. typhimurium TA98 upon exposure to a phenanthrene, b 1-methylphenanthrene, c 2-methylphenanthrene, d 3-methylphenanthrene and e 9-methylphenanthrene in absence (black bar) and presence (white bar) of 5\% S9-mix. Bars represent means and vertical bars indicate the standard deviation of the mean $(n=3)$. The dotted horizontal line indicates threefold increase that is considered the threshold for concluding on the positive outcome for (c)

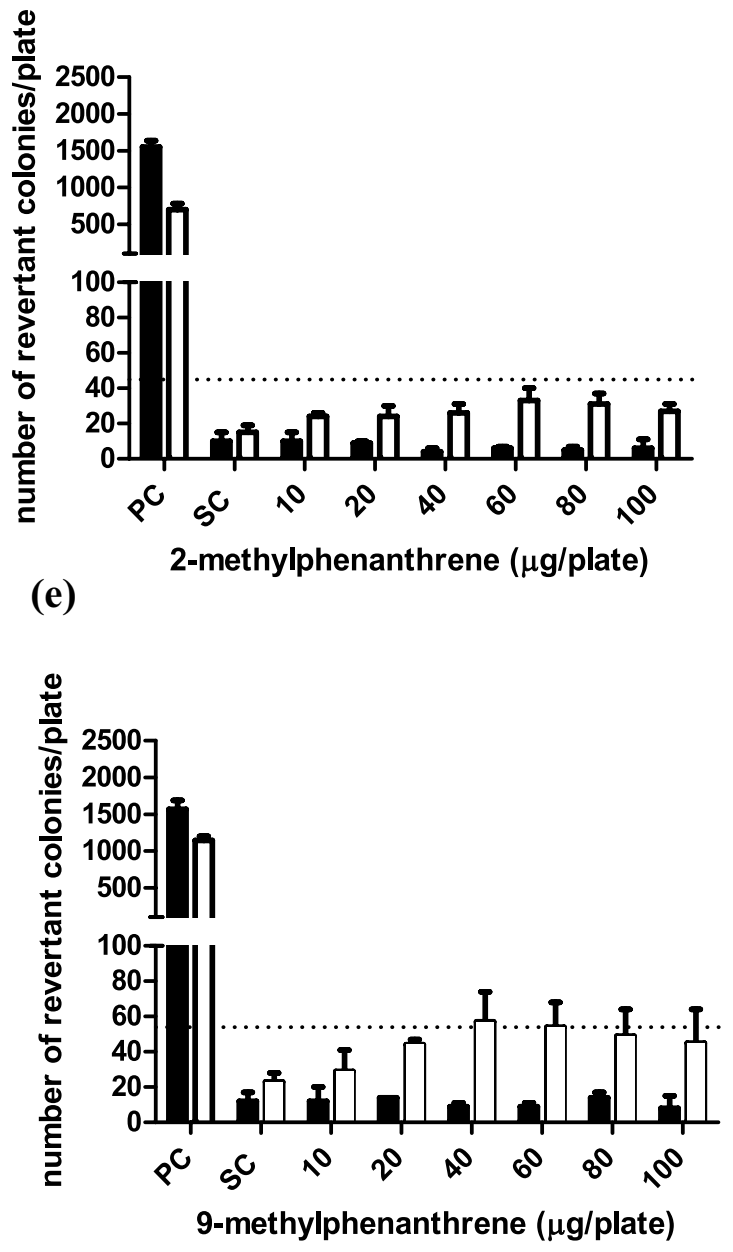

mutagenicity. In the absence of S9 mix, the test doses of 3-methylphenanthrene showed cytotoxicity so no data are presented. The results of at least four analyzable doses that were non-cytotoxic are presented in Table S2 in the supplementary material 1 showing negative results. $P C$ positive control, $1 \mu \mathrm{g}$ /plate $2 \mathrm{AA}$ with $\mathrm{S} 9$-mix and $10 \mu \mathrm{g} /$ plate NF without S9-mix. SC solvent control, DMSO with and without S9-mix 
(a)

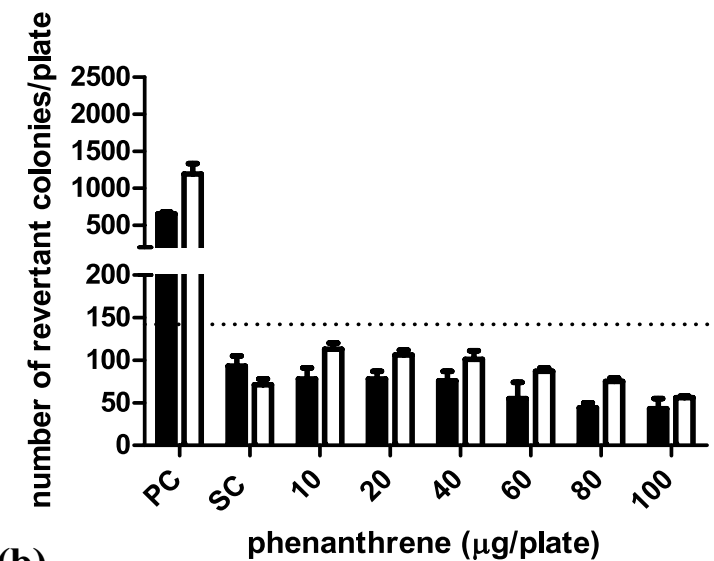

(b)

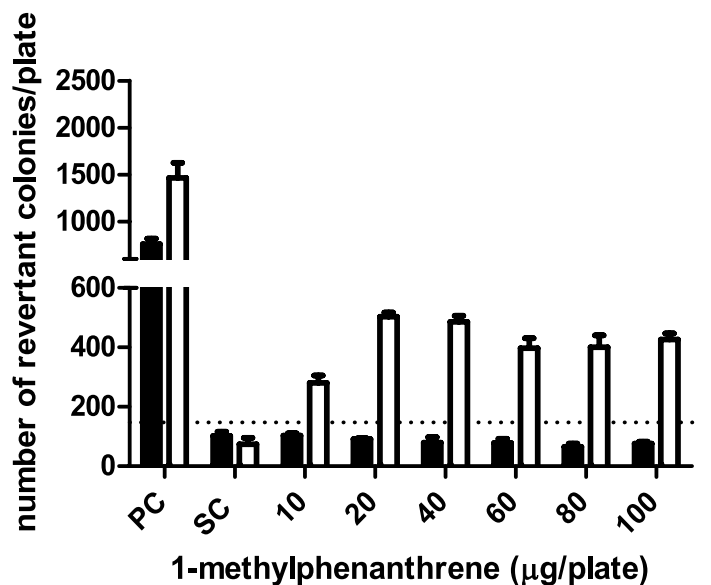

(d)

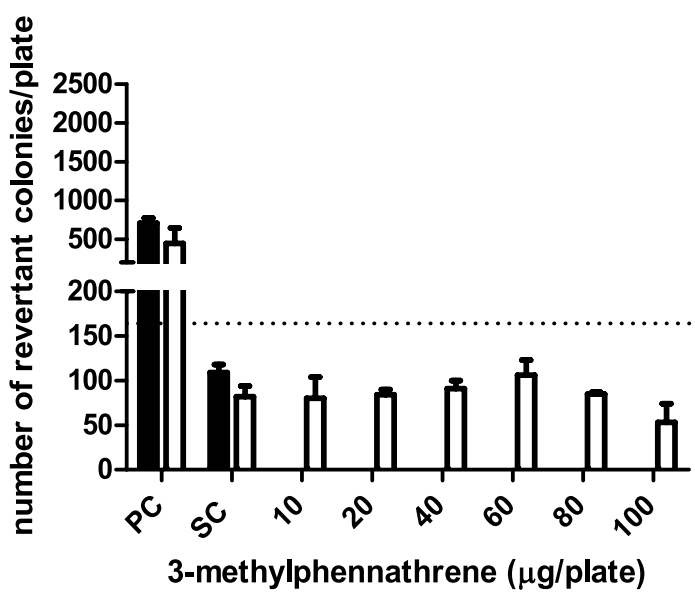

Fig. 6 Number of revertants in S. typhimurium TA100 upon exposure to a phenanthrene b 1-methylphenanthrene, c 2-methylphenanthrene, d 3-methylphenanthrene and $\mathbf{e} 9$-methylphenanthrene in the absence (black bar) and presence (white bar) of 5\% S9-mix. The dotted horizontal line indicates twofold increase that is considered the threshold for concluding on the positive outcome for mutagenicity. Bar represents means and vertical bars are standard deviation of the mean (c)

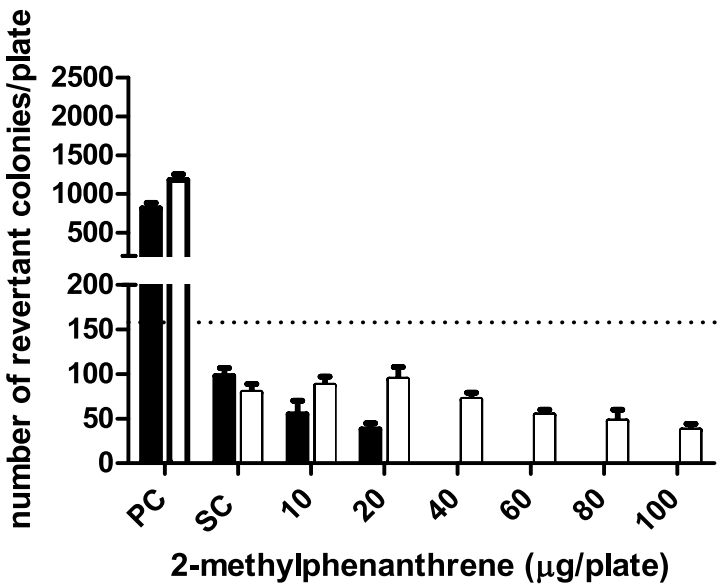

(e)

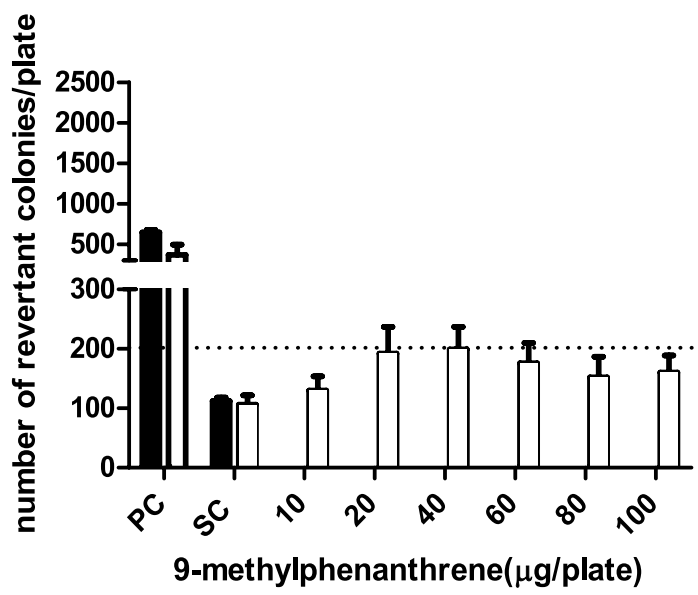

$(n=3)$. In the absence of the S9 mix, the test doses of 2-methylphenanthrene, 3-methylphenanthrene and 9-methylphenanthrene showed cytotoxicity so no data are presented. The results of at least four analyzable doses that were non-cytotoxic are presented in Table S3 in the supplementary material 1 showing negative results. $P C$ positive control, $5 \mu \mathrm{g} /$ plate $2 \mathrm{AA}$ with S9-mix and $650 \mu \mathrm{g} /$ plate MMS without S9-mix. SC solvent control, DMSO with and without S9-mix 
thus obtained show that the metabolism of alkylated phenanthrenes by both RLM and HLM becomes less efficient with elongation of the alkyl chain, especially when the side chain carbon number was more than 3 . No metabolic conversion was detected when the side chain carbon number was 12 .

\section{Mutagenicity of phenanthrene and its methylated substituents in the Ames test}

The reverse mutation assay (Ames test) was used to assess the effect of methylation of phenanthrene on its mutagenicity toward S. typhimurium tester strains TA98 and TA100. The model compounds used for these studies were nonsubstituted phenanthrene, phenanthrene with the methyl substitution at the $\mathrm{C} 1$ or $\mathrm{C} 9$ position generating a "fake" bay region and at $\mathrm{C} 2$ and $\mathrm{C} 3$ for which this was not the case. No precipitation was observed on the plates at the tested concentrations under the microscope. None of the test compounds showed mutagenic potential in both tester strains in the absence of S9 metabolic system (Figs. 5 and 6). Figures 5 and 6 also present the number of (His ${ }^{+}$) revertant colonies per plate induced in the presence of an $\mathrm{S} 9$ metabolic system by (a) phenanthrene, (b) 1-methylphenanthrene, (c) 2-methylphenanthrene, (d) 3-methylphenanthrene and (e) 9-methylphenanthrene for tester strain TA98 and TA100, respectively. No increases in the number of revertants were observed in TA 98 and TA100 upon exposure to phenanthrene in the presence of an S9 metabolic system. The observed increases in the number of revertants upon exposure to 1-methylphenanthrene in the presence of an $\mathrm{S} 9$ metabolic activation system was up to 13- and 6.8-fold compared to the concurrent solvent control for tester strain TA98 and TA100, respectively. In the presence of S9 metabolic activation, for both 2-methylphenanthrene and 3-methylphenanthrene, a slight dose response with 2.2-fold increase was observed in TA98; however, this was lower than the 3-fold increase required to conclude on a positive response that is

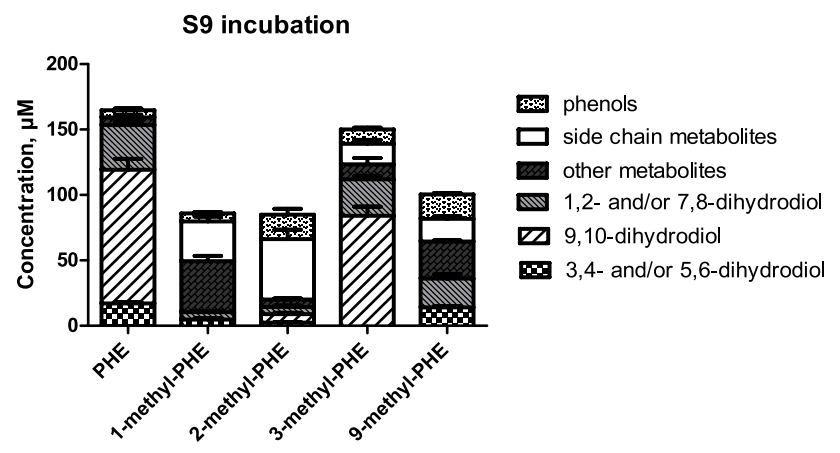

Fig. 7 Concentration of the metabolites formed in S9 incubation with phenanthrene and four of its methyl-substituted analogs. Each bar represents experimental means and vertical bars are standard errors of the mean $(n=3)$ biologically relevant (Levy et al. 2019) and no increased response was found in TA100 for either compound. Therefore, 2-methyl- and 3-methyl- phenanthrene were considered to be non-mutagenic in both TA98 and TA100. 2.5-Fold and 1.9-fold increases in the number of revertants compared to the concurrent solvent control were observed for 9-methylphenanthrene in TA98 and TA100, respectively, which were both lower than the 3-fold and 2-fold criteria. Close inspection of the corresponding solvent control data revealed that this was caused by the relatively high number of revertants in the concurrent solvent controls in the TA98 and TA100 assay of 9-methylphenanthrene, e.g., $23 \pm 5$ for the solvent control of 9-methylphenanthrene compared to the historical dataset with a value of $18 \pm 6$ for TA98 (Table S1). This implies that relative to the historical control data the increase in the number of revertants of 9-methyl-phenanthrene are 3.2-fold and 2.0-fold and are considered to be biologically relevant and indicative of mutagenicity.

\section{S9 mediated metabolism of phenanthrene and its methylated analogs}

To obtain insight into the metabolic activation of the four selected methyl-substituted phenanthrenes tested in the reverse mutation assay, exposure mixtures similar to those of the Ames test were analyzed by UPLC and further by GC-MS/MS for the unidentifiable metabolites. Figure 7 shows the metabolite patterns and quantification from the incubations with phenanthrene and its four methylated phenanthrenes (tested at $1000 \mu \mathrm{M}$ a concentration being equivalent to the highest dose of $100 \mu \mathrm{g}$ tested in the Ames test). Similarly to the results obtained with the microsomal incubations, metabolism of methylated phenanthrenes mediated by aroclor 1254 induced S9-mix generated dihydrodiols, alcohols and phenols (Figure S1 in supplementary material 1). This reveals that similar to what was already observed for the microsomal incubations, also for the S9 incubations the presence of the alkyl substituent shifts the metabolism of phenanthrene in favor of side chain oxidation at the cost of aromatic oxidation. Besides, an additional type of metabolite (reflected by a peak marked with an asterisk in Figure S1 supplementary material 1) was identified as hydroxymethylhydroxy-phenanthrene representing a further metabolite of hydroxymethyl-phenanthrene with a molecular ion at $\mathrm{m} / \mathrm{z}$ 226 measured by GC-MS/MS.

Three major metabolites of phenanthrene observed in the chromatogram (Figure $\mathrm{S} 1$ in supplementary material 1) of the $S 9$ incubation were identified as 3,4-dihydrodiol, 9,10-dihydrodiol and 1,2-dihydrodiol accounting for $10.5 \%$, $62.1 \%$ and $20.7 \%$ of the total metabolite formation of phenanthrene, respectively. Formation of 1,2- and/or 7,8-dihydrodiols was found in incubations with all tested monomethylated phenanthrenes. Variation in the formation of 3,4- and/ 
or 5,6-dihydrodiols and 9,10-dihydrodiol between mutagenic phenanthrenes (1-methyl- and 9 methyl-phenanthrene) and non-mutagenic phenanthrenes (2-methyl- and 3-methylphenanthrene) was noticed (Fig. 7). Formation of 9,10-dihydrodiol was only found in the incubations with 2-methyl and 3 -methylphenanthrene at levels amounting to $7.7 \%$ and $57.7 \%$ of the metabolites formed, respectively. In the S9 incubations, 3,4- and/or 5,6-dihydrodiols were formed at levels amounting to $5.5 \%$ and $12.5 \%$ of the total metabolite formation for 1-methyl- and 9-methyl-phenanthrene, and at $2.2 \%$ and $0 \%$ of the total metabolite formation for 2-methyl and 3-methylphenanthrene, respectively.

\section{Discussion}

The consumption of food contaminated with PPAHs from petroleum-derived products or environmental sources may be of concern to human health. The hazards of PPAHs are expected to be related to bioactivation. Since data on the metabolic fate of most PPAHs, in particular alkylated PAHs, are lacking, the microsomal oxidative metabolism, likely mediated by cytochrome P450 enzymes, of phenanthrenes with various types of alkylation was studied to investigate the effect of the alkyl substitution. It was hypothesized that alkylation of phenanthrenes would shift metabolism to alkyl side chain oxidation at the cost of aromatic ring oxidation when compared to non-alkylated phenanthrene. This metabolic shift would be expected to lower the chances of bioactivation to metabolites such as possibly mutagenic dihydrodiols and developmental toxicity related phenols. In line with the hypothesis, the side chain hydroxylated metabolites of alkylated phenanthrenes were 30.9- to 3.5fold more efficiently formed than aromatic ring oxidation metabolites by both RLM and HLM. The overall metabolism of phenanthrene with an alkyl chain with more than six carbon atoms was strongly reduced and metabolism was even absent in the case of 1-n-dodecylphenanthrene (C12). This observation may be best ascribed to possible steric hindrance by the longer alkyl chains hampering binding to the active site of the cytochrome P450 enzymes. Furthermore, metabolic oxidation primarily happened on the carbon atom at the benzylic position in case of 2-ethyl-, 9-ethyl-, and 10-methyl-9-ethyl-phenanthrenes, similar to the results obtained by microsomal incubations of 1-ethyl- and 2-ethylnaphthalene (Wang et al. 2020). Side chain oxidation of alkylated phenanthrenes was also observed in other metabolic studies. Hydroxymethyl-phenanthrenes were detected as primary metabolites in a metabolism study with rat $S 9$ incubations of 1-methyl-, 2-methyl, 3-methyl-, 4-methyland 9-methyl-phenanthrene (LaVoie et al. 1981). Side chain hydroxylation metabolites were also detected in incubations of 1-methylphenanthrene and 9-ethylphenanthrene with human hepatoma (HepG2) cells (Huang et al. 2017).

Considering that the metabolic shift from aromatic ring to side chain oxidation may reflect a shift from bioactivation to detoxification, data on the mutagenicity and tumorigenicity of these alkylated PAHs are of interest. However, data on the mutagenicity and the tumorigenicity of phenanthrene and alkyl-substituted phenanthrenes are scarce. Neither phenanthrene nor its dihydrodiol metabolites were found to be mutagenic toward TA98 and TA100 tester strain of S. typhimurium (Bucker et al. 1979; Wood et al. 1979). This is in line with the observation in the present study that phenanthrene tested negative for mutagenicity in both tester strains TA98 and TA100. In the present study, 1-methylphenanthrene showed 13 -fold and 6.8-fold increase in the number of revertants compared to the concurrent solvent control when tested in the presence of metabolic activation in TA98 and TA100, respectively. Comparable studies showed a 3.2-fold and 6.0-fold increase in TA98 and TA100, respectively (LaVoie et al. 1983), and an 8.4-fold and 4.1-fold increase in the same strains (Katarzyna Rudnicka et al. 2013). In addition, 9-methylphenanthrene, the
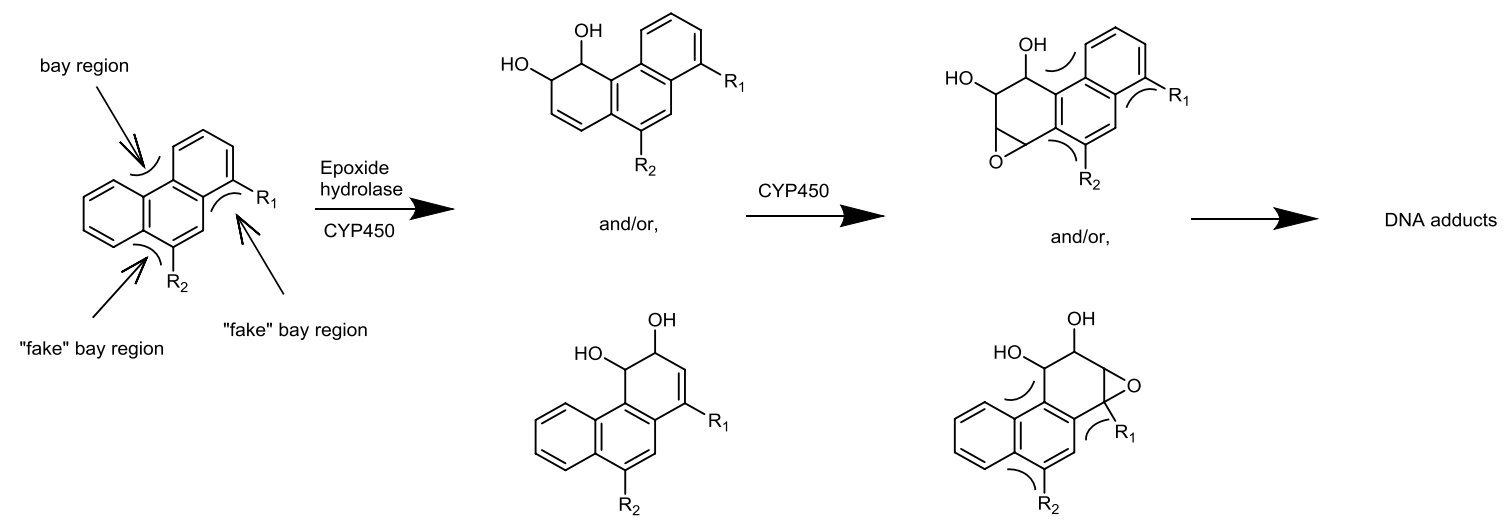

Fig. 8 Possible metabolic pathway toward bay region dihydrodiol-epoxide formation for 1-methyl- $\left(\mathrm{R}_{1}=\mathrm{CH}_{3}\right)$ and 9-methyl- $\left(\mathrm{R}_{2}=\mathrm{CH}_{3}\right)$ phenanthrene 
other methyl-substituted phenanthrene that tested positive with 3.2-fold (TA98) and 2.0-fold (TA100) increase relative to historical controls in the present study, was previously reported to be mutagenic as reflected by a 3.0-fold increase in revertants in TA100 while testing negative (only 1.3-fold increase) in TA98 (LaVoie et al. 1983).

Considering that the possible metabolic pathway underlying mutagenicity of 1-methyl- and 9-methyl-phenanthrene could be a dihydrodiol-epoxide pathway (Fig. 8), it can be suggested that the observed mutagenicity of 1-methyl- and 9-methyl-phenanthrene may be associated with regio- and stereo- selectivity for the possible dihydrodiol-epoxide bioactivation following the introduction of the methyl substitution. The formation of an additional bay region-like structural motif, described as "fake" bay region (Fig. 8) upon introduction of a methyl substituent at the $\mathrm{C} 1$ or $\mathrm{C} 9$ position of phenanthrene, may play a role in the observed mutagenicity. It is also of interest to note that it has been suggested before that the formation of 3,4- and/ or 5,6- dihydrodiol metabolites likely reflects the bioactivation to mutagenic metabolites (LaVoie et al. 1981). This would be in line with the suggestion that methyl substitution at or near the K-region (9,10-position) of phenanthrene at its peri positions (Fig. 1) would favor 3,4- and/or 5,6-dihydrodiol formation and mutagenicity (Fig. 5 and 6) (LaVoie et al. 1983). Indeed for 9-methyl- and 1-methylphenanthrene, formation of 3,4- and/or 5,6-dihydrodiol was observed, albeit to a level somewhat lower than that observed for phenanthrene itself which was tested negative for mutagenicity. Nevertheless, given the fact that methyl substitution adjacent to the K-region in 1-methyland 9-methyl phenanthrene forms a "fake" bay region, it implies that 3,4- and/or 5,6-dihydrodiol formation provides increased chances of formation of a bay-region dihydrodiol-epoxide (Fig. 8). However, in phenanthrene this 3,4and/or 5,6-dihydrodiol formation would not result in a bay region dihydrodiol-epoxide.

Given these mutagenicity results, it is also important to consider that neither phenanthrene nor its monomethylated analogs, including 2-methyl- and 3-methyl-phenanthrene but also 1-methyl- and 9-methyl-phenanthrene, were found to be active tumor initiators in mouse skin painting studies (Buening et al. 1979; LaVoie et al. 1981). This lack of tumor-initiating activity of methyl-substituted phenanthrenes might be due to a relatively lower tumor inducing potency of the formed DNA adducts compared to for example the dihydrodiol-epoxide metabolites of benzo[a]pyrene.

Finally, it is also of interest that some authors have proposed a role for side chain hydroxylated metabolites in alkyl-substituted PAHs to play a role in their mutagenicity upon their further bioactivation by sulfotransferases to unstable DNA reactive sulfate metabolites (Huang et al. 2017). Whether for 1-methyl- and 9-methyl-phenanthrene also their side chain hydroxy metabolites play such a role remains to be investigated.

The current risk assessment of PPAHs that may be present in consumer products is based on read across to metabolic activation and formation of DNA reactive metabolites of naked PAHs due to the lack of data on PPAH themselves (Baird et al. 2007; EFSA 2012; Wickliffe et al. 2014). The results of the present study provide insight in the effect of alkylation on the oxidative metabolism of phenanthrene, and also provide kinetic parameters that may turn out to be of use for future physiologically based kinetic (PBK) models to extrapolate toxicity data obtained in vitro to in vivo taking kinetics into account. The PBK models together with in vitro concentration-response data could provide the basis for a new approach methodology (NAM) for predicting the in vivo toxicity of alkyl-substituted aromatics that may be present in petroleum-derived products, in line with the proof of principle predicting the developmental toxicity of benzo[a]pyrene by this in vitro-in silico approach (Wang et al. 2021).

Phenanthrene is the smallest PAH with a bay region, but lacks genotoxic and carcinogenic properties observed for some bay-region PAHs with a higher number of aromatic rings, such as benzo[a]pyrene. It would be of interest for future studies to investigate whether alkylation causes similar shifts in metabolic oxidation for PAHs and PPAHs with more fused aromatic rings and in what way this influences their toxicity, as observed with naphthalene (Wang et al. 2020) and with phenanthrene in the present study. The results of the present study clearly show that the mutagenic effect depends on the site of alkylation on PAHs.

Taking all together, it is concluded that alkylation of PAHs favors alkyl chain oxidation at the cost of aromatic oxidation. Especially methyl substitution of phenanthrene adjacent to its K-region such as in 1-methyl- and 9-methyl-phenanthrene converted phenanthrene into mutagens toward S. typhimurium TA98 and TA100. The position of the alkylation affects the metabolism and resulting mutagenicity of phenanthrene with the mutagenicity increasing in cases where the alkyl substituent creates an additional bay region-like structural motif, in spite of the extra possibilities for side chain oxidation.

Supplementary Information The online version contains supplementary material available at https://doi.org/10.1007/s00204-022-03239-9.

Funding This work was financially supported by Concawe (No. 201700093) in Belgium, and by a grant from China Scholarship Council from China (No. 201807720073) to Danlei Wang. Part of this work was supported by Operationeel Programma Kansen voor West II (EFRO) (project no KVW- 00181). 


\section{Declarations}

Conflict of interest Danlei Wang, Viktoria Schramm, Jeroen Pool, Eleni Pardali, Annemarijn Brandenburg, Ivonne M.C.M. Rietjens and Peter J. Boogaard declare that they have no conflict of interest.

Open Access This article is licensed under a Creative Commons Attribution 4.0 International License, which permits use, sharing, adaptation, distribution and reproduction in any medium or format, as long as you give appropriate credit to the original author(s) and the source, provide a link to the Creative Commons licence, and indicate if changes were made. The images or other third party material in this article are included in the article's Creative Commons licence, unless indicated otherwise in a credit line to the material. If material is not included in the article's Creative Commons licence and your intended use is not permitted by statutory regulation or exceeds the permitted use, you will need to obtain permission directly from the copyright holder. To view a copy of this licence, visit http://creativecommons.org/licenses/by/4.0/.

\section{References}

Baird SJS, Bailey EA, Vorhees DJ (2007) Evaluating human risk from exposure to alkylated PAHs in an aquatic system. Hum Ecol Risk Assess 13(2):322-338. https://doi.org/10.1080/108070307012262 77

Bao ZP, Yang SK (1991) Liquid-chromatographic separation of isomeric phenanthrols on monomeric and polymeric C18 columns. J Chromatogr 536(1-2):245-249. https://doi.org/10.1016/S00219673(01)89256-6

Bayer U (1978) In vivo induction of sister chromatid exchanges by three polyaromatic hydrocarbons polynuclear aromatic hydrocarbons, 1st edn. Raven Press, New York, pp 423-428

Boogaard PJ (2012) Chapter 3F biomonitoring of exposure to polycyclic aromatic hydrocarbons biomarkers and human biomonitoring, 1st edn. The Royal Society of Chemistry, London, pp 338-359

Bucker M, Glatt HR, Platt KL et al (1979) Mutagenicity of phenanthrene and phenanthrene K-region derivatives. Mutat Res 66(4):337-348. https://doi.org/10.1016/0165-1218(79)90044-2

Buening MK, Levin W, Karle JM, Yagi H, Jerina DM, Conney AH (1979) Tumorigenicity of bay-region epoxides and other derivatives of chrysene and phenanthrene in newborn mice. Cancer Res 39(12):5063-5068

Carrillo JC, van der Wiel A, Danneels D, Kral O, Boogaard PJ (2019) The selective determination of potentially carcinogenic polycyclic aromatic compounds in lubricant base oils by the DMSO extraction method IP346 and its correlation to mouse skin painting carcinogenicity assays. Regul Toxicol Pharmacol 106:316-333. https://doi.org/10.1016/j.yrtph.2019.05.012

Chaturapit S, Holder GM (1978) Studies on the hepatic microsomal metabolism of (14C) phenanthrene. Biochem Pharmacol 27(14):1865-1871. https://doi.org/10.1016/0006-2952(78) 90034-5

EFSA (2008) Polycyclic aromatic hydrocarbons in food scientific opinion of the panel on contaminants in the food chain. EFSA J 6(8):724. https://doi.org/10.2903/j.efsa.2008.724

EFSA (2012) Scientific Opinion on Mineral Oil Hydrocarbons in Food. EFSA J 10(6):2704. https://doi.org/10.2903/j.efsa.2012.2704

Fengler R, Gruber L (2020) Mineral oil migration from paper-based packaging into food, investigated by means of food simulants and model substances. Food Addit Contam. https://doi.org/10.1080/ 19440049.2020.1714750

Fernando H, Ju H, Kakumanu R et al (2019) Distribution of petrogenic polycyclic aromatic hydrocarbons (PAHs) in seafood following
Deepwater Horizon oil spill. Mar Pollut Bull 145:200-207. https://doi.org/10.1016/j.marpolbul.2019.05.015

Grob K (2018) Mineral oil hydrocarbons in food: a review. Food Addit Contam 35(9):1845-1860. https://doi.org/10.1080/19440049. 2018.1488185

Huang M, Mesaros C, Hackfeld LC, Hodge RP, Blair IA, Penning TM (2017) Potential metabolic activation of representative alkylated polycyclic aromatic hydrocarbons 1-methylphenanthrene and 9-ethylphenanthrene associated with the deepwater horizon oil spill in human hepatoma (HepG2) cells. Chem Res Toxicol 30(12):2140-2150. https://doi.org/10.1021/acs.chemrestox.7b002 32

IARC (2010) Some non-heterocyclic polycyclic aromatic hydrocarbons and some related exposures, vol 92. World Health Organization, Lyon France

Jacob J, Raab G, Soballa V et al (1996) Cytochrome P450-mediated activation of phenanthrene in genetically engineered V79 Chinese hamster cells. Environ Toxicol Pharmacol 1(1):1-11. https://doi. org/10.1016/1382-6689(95)00003-8

Katarzyna Rudnicka ST, Budzikur KA, Mielżyńska-Švach D, Jakimiuk E, Chachaj A, Góra M, Żelazna K, Łuczyński MK (2013) Assessment of mutagenic activity of methyl- and phenylphenanthrenes based on Salmonella test and micronucleus test. Environ Biotechnol 9(2):65-71. https://doi.org/10.14799/ebms193

LaVoie EJ, Tulley-Freiler L, Bedenko V, Hoffman D (1981) Mutagenicity, tumor-initiating activity, and metabolism of methylphenanthrenes. Cancer Res 41(9 Pt 1):3441-3447

LaVoie EJ, Tulley-Freiler L, Bedenko V, Hoffmann D (1983) Mutagenicity of substituted phenanthrenes in Salmonella typhimurium. Mutat Res 116(2):91-102. https://doi.org/10.1016/0165-1218(83) 90100-3

Lehr RE, Kumar S, Levin W et al (1985) The Bay Region theory of polycyclic aromatic hydrocarbon carcinogenesis. ACS Symp Ser 283:63-84

Levy DD, Zeiger E, Escobar PA et al (2019) Recommended criteria for the evaluation of bacterial mutagenicity data (Ames test). Mutat Res Genet Toxicol Environ Mutagen 848:403074. https://doi.org/ 10.1016/j.mrgentox.2019.07.004

Li D, Han YL, Meng XL et al (2010) Effect of regular organic solvents on cytochrome p450-mediated metabolic activities in rat liver microsomes. Drug Metab Dispos 38(11):1922-1925. https:// doi.org/10.1124/dmd.110.033894

NIST (2012) Standard Reference Material (SRM) 2779, Gulf of Mexico Crude Oil. National Institute of Standards and Technology, Gaithersburg, MD, pp 1-7

Pirow R, Blume A, Hellwig N et al (2019) Mineral oil in food, cosmetic products, and in products regulated by other legislations. Crit Rev Toxicol 49(9):742-789. https://doi.org/10.1080/10408 444.2019.1694862

Pulster EL, Gracia A, Armenteros M et al (2020) A first comprehensive baseline of hydrocarbon pollution in Gulf of Mexico fishes. Sci Rep UK. https://doi.org/10.1038/s41598-020-62944-6

Schober W, Pusch G, Oeder S, Reindl H, Behrendt H, Buters JT (2010) Metabolic activation of phenanthrene by human and mouse cytochromes P450 and pharmacokinetics in CYP1A2 knockout mice. Chem Biol Interact 183(1):57-66. https://doi.org/10.1016/j. cbi.2009.09.008

Shou M, Korzekwa KR, Krausz KW, Crespi CL, Gonzalez FJ, Gelboin HV (1994) Regio- and stereo-selective metabolism of phenanthrene by twelve cDNA-expressed human, rodent, and rabbit cytochromes P-450. Cancer Lett 83(1-2):305-313. https://doi.org/ 10.1016/0304-3835(94)90334-4

Sims P (1970) Qualitative and quantitative studies on the metabolism of a series of aromatic hydrocarbons by rat-liver preparations. Biochem Pharmacol 19(3):795-818. https://doi.org/10.1016/ 0006-2952(70)90243-1 
Tsang W-S, Griffin GW (1979) The "Bay-Region" theory of carcinogenic activity; application of perturbational molecular orbital theory. In: Tsang W-S, Griffin GW (eds) Metabolic activation of polynuclear aromatic hydrocarbons. Elsevier, Amsterdam, pp 73-85

Van Heyst A, Vanlancker M, Vercammen J et al (2018) Analysis of mineral oil in food: results of a Belgian market survey. Food Addit Contam 35(10):2062-2075. https://doi.org/10.1080/19440049. 2018.1512758

Wang D, Bruyneel B, Kamelia L, Wesseling S, Rietjens IMCM, Boogaard PJ (2020) In vitro metabolism of naphthalene and its alkylated congeners by human and rat liver microsomes via alkyl side chain or aromatic oxidation. Chem Biol Interact 315:108905. https://doi.org/10.1016/j.cbi.2019.108905

Wang D, Rietdijk MH, Kamelia L, Boogaard PJ, Rietjens IMCM (2021) Predicting the in vivo developmental toxicity of benzo[a] pyrene $(\mathrm{BaP})$ in rats by an in vitro-in silico approach. Arch Toxicol. https://doi.org/10.1007/s00204-021-03128-7

Whalen DL, Ross AM, Yagi H, Karle JM, Jerina DM (1978) Stereoelectronic factors in the solvolysis of bay region diol epoxides of polycyclic aromatic hydrocarbons. J Am Chem Soc 100(16):52185221. https://doi.org/10.1021/ja00484a058

Wickliffe J, Overton E, Frickel S et al (2014) Evaluation of polycyclic aromatic hydrocarbons using analytical methods, toxicology, and risk assessment research: seafood safety after a petroleum spill as an example. Environ Health Perspect 122(1):6-9. https://doi.org/ 10.1289/ehp.1306724

Wood AW, Chang RL, Levin W et al (1979) Mutagenicity and tumorigenicity of phenanthrene and chrysene epoxides and diol epoxides. Cancer Res 39(10):4069-4077

Ylitalo GM, Krahn MM, Dickhoff WW et al (2012) Federal seafood safety response to the Deepwater Horizon oil spill. Proc Natl Acad Sci USA 109(50):20274-20279. https://doi.org/10.1073/ pnas. 1108886109

Publisher's Note Springer Nature remains neutral with regard to jurisdictional claims in published maps and institutional affiliations. 